\title{
Modified Whale Optimization Algorithm for Solar Cell and PV Module Parameter Identification
}

\author{
Xiaojia Ye, ${ }^{1}$ Wei Liu, ${ }^{2}$ Hong Li, ${ }^{3}$ Mingjing Wang, ${ }^{4}$ Chen Chi, ${ }^{5}$ Guoxi Liang $\mathbb{D},{ }^{6}$ \\ Huiling Chen $\mathbb{D}^{7}{ }^{7}$ and Hailong Huang ${ }^{7}$ \\ ${ }^{1}$ Shanghai Lixin University of Accounting and Finance, Shanghai 201209, China \\ ${ }^{2}$ Department of Mathematics and Statistics, York University, Canada \\ ${ }^{3}$ Wenzhou Vocational College of Science and Technology, Wenzhou, Zhejiang 325006, China \\ ${ }^{4}$ Institute of Research and Development, Duy Tan University, Da Nang 550000, Vietnam \\ ${ }^{5}$ Oujiang College, Wenzhou University, Wenzhou 325035, Zhejiang, China \\ ${ }^{6}$ Department of Information Technology, Wenzhou Polytechnic, Wenzhou 325035, China \\ ${ }^{7}$ Department of Computer Science and Artificial Intelligence, Wenzhou University, Wenzhou 325035, Zhejiang, China \\ Correspondence should be addressed to Chen Chi; chen_chi@189.cn, Huiling Chen; chenhuiling.jlu@gmail.com, and \\ Hailong Huang; hhl@wzu.edu.cn
}

Received 12 August 2020; Revised 4 January 2021; Accepted 8 January 2021; Published 23 February 2021

Academic Editor: Ramon Costa-Castelló

Copyright (c) 2021 Xiaojia Ye et al. This is an open access article distributed under the Creative Commons Attribution License, which permits unrestricted use, distribution, and reproduction in any medium, provided the original work is properly cited.

\begin{abstract}
The whale optimization algorithm (WOA) is a powerful swarm intelligence method which has been widely used in various fields such as parameter identification of solar cells and PV modules. In order to better balance the exploration and exploitation of WOA, we propose a novel modified WOA (MWOA) in which both the mutation strategy based on Levy flight and a local search mechanism of pattern search are introduced. On the one hand, Levy flight can make the algorithm get rid of the local optimum and avoid stagnation; thus, it is able to prevent the algorithm from losing diversity and to increase the global search capability. On the other hand, pattern search, a direct search method, has not only high convergence rate but also good stability, which can boost the local optimization ability of the WOA. Therefore, the combination of these two mechanisms can greatly improve the capability of WOA to obtain the best solution. In addition, MWOA may be employed to estimate parameters in single diode model (SDM), double diode model (DDM), and PV modules and to identify unknown parameters of two different types of PV modules under diverse light irradiance and temperature conditions. The analytical results demonstrate the validity and the practicality of MWOA for estimating parameters of solar cells and PV modules.
\end{abstract}

\section{Introduction}

Solar power generation is an emerging renewable energy technology, and photovoltaic system is a type of power generation system that uses the photovoltaic effect of solar cell semiconductor materials to convert sunlight radiation energy into electricity. Moreover, due to its advantages such as safety, reliability, being nonpolluting, and unlimitedness by the distribution of resources, photovoltaic system has been widely used in many fields $[1,2]$. Thus, the research on the features of solar cells and photovoltaic modules, which are important components of PV systems, is an ongoing focus of attention. The properties of solar cells and PV modules are usually determined by the current-voltage curve. However, because of its strong nonlinear characteristics, just the current-voltage curve is not sufficient to analyze the changes in the characteristics of the new battery with temperature, light intensity, and so on. Therefore, the modeling and the parameter searching of solar cells and PV systems is one of the hot topics in the current study.

A suitable model and an appropriate parameter extraction method are essential for the simulation, design, evaluation, control, and optimization of PV systems [3]. Currently the models used to characterize the cells include 
SDM, DDM, and TDM with SDM and DDM being the most commonly used [4]. The methods for extracting parameters can be roughly classified as analytical method [5-7], method with Lambert W function $[8,9]$, method of constructing or using special function $[10,11]$, or method with swarm intelligence algorithm. Among these methods, swarm intelligence algorithms have received great attention in recent years, and, compared with traditional methods, the swarm intelligence algorithm has a simple structure, fewer parameters, and high efficiency, such as particle swarm optimization (PSO) [12], differential evolution (DE) [13], naked mole-rat algorithm (NMR) [14], Harris hawks optimizer (HHO) [15], and slime mould algorithm (SMA) [16]. Furthermore, a large number of studies have focused on using such methods or their variants for modeling and parameter extraction of solar cells and PV modules [17-24].

Messaoud et al. [25] used Salp Swarm Algorithm (SSA) for the parameter estimation in SDM and DDM and applied it to three different PV parameter identification problems. Abbassi et al. [26] proposed an efficient method by using SSA to extract DDM parameters of PV cells and an opposition-based learning modified SSA (OLMSSA) [27] for identification of PV cells and modules. Pourmousa et al. [28] considered an improved Lozi Map based Chaotic Optimization Algorithm (ILCOA) for parameter estimation of solar cells. Chen et al. [29] studied a new hybrid teaching learningbased artificial bee colony (TLABC) to obtain solar PV parameter estimates, which combined a teaching-learningbased optimization algorithm (TLBO) with an artificial bee colony (ABC). Li et al. [30] suggested an improved teachinglearning-based optimization (ITLBO) algorithm for estimating the parameters of SDM, DDM, and three photovoltaic modules. Liang et al. [31] proposed an evolutionary multitask optimization algorithm to simultaneously extract parameters from several different PV models. Li et al. [32] gave an enhanced adaptive differential evolution algorithm for PV parameter extraction, which performed well for SDM, DDM, and PV modules. Li and Wu [33] discussed a memetic adaptive differential evolution (MADE) algorithm for the parameter estimation of photovoltaic models. Chen et al. [34] considered a hybrid metaheuristic called biogeography-based heterogeneous cuckoo search (BHCS) algorithm in order to enhance the parameter estimation capability of solar PV models. Yousri et al. [35] proposed novel chaotic heterogeneous comprehensive learning PSO variants, in which the chaotic heterogeneous comprehensive learning PSO is combined with ten different chaos diagrams to adjust model parameters, and they applied this new approach for parameter extraction of PV models. Liang et al. [36] studied a classified perturbation mutation based particle swarm optimization algorithm for the extraction of parameters from five different PV models. Lin [37] proposed an optimization algorithm based on niche particle swarm optimization in parallel computing (NPSOPC) to determine the parameters of photovoltaic models. Jian et al. [38] suggested a logistic chaotic JAYA algorithm (LCJAYA) for parameter identification of PV cells and modules. Zhang [39] discovered a backtracking search algorithm with Levy flight (LGBSA) to estimate parameters in three different PV models. Zhang et al. [40] suggested an orthogonal moth-fighting optimization algorithm (MFO) with local search (NMSOLMFO) to identify the parameters of PV cell models.

Traditional swarm intelligence algorithms such as PSO and $\mathrm{ABC}$ have already performed well in this field. However, the problem of photovoltaic system parameter evaluation is a multimodal problem; hence, there is no way to obtain the global optimal solution due to prematurity of some basic algorithms. According to the principle of no free lunch, it is still necessary to develop an efficient optimizer for accurately extracting model parameters. Subsequently, WOA is considered because of its simple structure, few parameters, strong search ability, and easy implementation; it has been successfully applied in various fields such as reactive scheduling [41], neural networks [42], image segmentation [43] and feature selection [44], and wind speed prediction [45]. Besides, many scholars are also committed to improving its existing shortcomings to develop more effective optimizers; for example, Salgotra et al. [46] utilized opposition-based learning, exponentially decreasing parameters, and elimination or reinitialization of worst particles to enhance the basic WOA. Chen et al. [47] introduced random replacement and double adaptive weight to speed up convergence and improve exploratory convergence direction. Three new versions of the WOA were designed by using nonlinear convergence factors and introducing the concept of chaos in the initialization stage as well as in the calculation of internal weights [48]. Ling et al. [49] used the Levy flight trajectory to improve the disadvantage that the underlying WOA is premature and prone to fall into local optimum. Zhou et al. [50] improved WOA by teaching learning-based algorithm and simplex method and applied it to train multilayer perceptron neural network. Niu et al. [51] developed an optimizer by dynamically combining the conventional WOA with the Levenberg-Marquardt (LM) algorithm and applied it to the alignment star sensor. Luo et al. [52] utilized a ranking-based mutation operation to modify WOA for identify the infinite impulse system. Moreover, WOA and its variants are also utilized for identifying parameters of solar cells and PV modules. Long et al. [53] introduced logistic models and a new refractivelearning strategy based on the refractive principle of light into WOA and proposed a new approach (RLWOA) for parameter estimation in PV models. Xiong et al. [54] developed two prey searching mechanisms to balance exploitation and exploration and proved that the method can effectively avoid premature phenomena for the accurate extraction of parameters from different PV models. Elazab et al. [55] studied a novel whale optimization algorithm for the parameter estimation of SDM, DDM, and TDM of PV modules. Oliva et al. [56] suggested the chaotic whale optimization algorithm (CWOA) for estimating parameters of solar cells. Although a number of studies about WOA have been investigated for the parameter estimation of solar cells and photovoltaic modules, there is room for the improvement of WOA for this problem in both the accuracy and the speed of convergence. Consequently, it is believed that 
developing an improved optimizer based on WOA is necessary.

Like other swarm intelligence optimization algorithms, in the basic WOA, individuals in the population are constantly approaching the food source as the iteration progresses, causing a gradual loss of diversity in the population. However, the problem of evaluating the parameters of a photovoltaic system is a nonlinear, multimodal problem, so it is highly susceptible to local optimum due to the loss of population diversity when using WOA to solve the problem. Moreover, since WOA relies on a stochastic convergence factor to convert from exploration to exploitation, it also suffers from a weak local search capability. Accordingly, in this paper, a variation strategy using Levy flight and a local search mechanism (pattern search) is introduced to improve the performance of WOA. This modified whale optimization algorithm is referred to as MWOA. Specifically, as the iteration progresses, individuals in the population constantly approach the current optimal solution in order to find the source of food, which makes the search space solution gradually concentrate on the current optimal solution. However, the problem of PV model parameter evaluation is a multimodal problem. In other words, the current solution may not be the global optimum, and the population individuals may miss better solutions when they keep approaching it, which is often said to fall into the local optimum. However, the random step of Levy flight mainly consists of small strides with occasional large ones or long jumps, which allows Levy flight to generate a fraction of the solutions far from the current optimal solution so that MWOA can get rid of local optimum and avoid stagnation.

The random step length of Levy flight is mainly composed of small step lengths, and occasionally there will be large step lengths or long jumps, which allows Levy flight to generate some solutions far away from the current optimal solution [57]. To a certain extent, this can guarantee the quality of the population during the entire evolution process, thereby helping MWOA to get rid of the local optimum and avoid stagnation. Consequently, it can be considered that the introduction of Levy flight can prevent the algorithm from losing its overall diversity and enhance global search capabilities. Since the pattern search mechanism usually searches around the current best solution, it has high stability and good convergence rate. The pattern search algorithm is considered in order to be able to enhance the local search capability of the basic WOA and improve the optimization accuracy. The addition of the two mechanisms has improved the optimization of WOA in terms of its ability to seek out the best solution and the balance between exploration and exploitation of the algorithm. Moreover, MWOA can be employed to identify unknown parameters of SDM, DDM, and PV modules. For two different types of PV modules, MWOA can identify their parameters under various temperature and light irradiance conditions, which demonstrates its validity and practicality.
The structure of this paper is as follows. Section 2 provides the problem formulation of SDM, DDM, and PV module models. MWOA is proposed in detail in Section 3. The experiments are displayed in Section 4. The conclusions are delivered in Section 5.

\section{Problem Definition}

The problem of parameter identification in solar cells and photovoltaic systems is to determine the parameters in the model by using the measured current and voltage values. However, the equivalence model to simulate the nonlinear current-voltage relationship of solar cells and PV modules mainly includes SDM and DDM, while SDM is commonly adopted in application.

\subsection{Solar Cell Model}

2.1.1. SDM. SDM is simple and its structure is shown in Figure 1. In Figure 1, there are several major physical parameters including photocurrent $I_{\mathrm{ph}}$, diode current $I_{d}$, shunt resistor $I_{\mathrm{sh}}$, shunt resistance $R_{\mathrm{sh}}$, series resistance $R_{S}$, and input current $I_{L}$. As seen from Figure 1, we can easily know the calculation of output current of SDM, whose expression is shown in the following equation:

$$
I_{L}=I_{\mathrm{ph}}-I_{d}-I_{\mathrm{sh}}
$$

According to the Shockley equation, diode current $I_{d}$ in equation (1) can be expressed as equation (2) while $I_{\text {sh }}$ can be given by equation (3):

$$
\begin{aligned}
& I_{d}=I_{\mathrm{sd}} \cdot\left[\exp \left(\frac{V_{L}+R_{S} \cdot I_{L}}{n \cdot V_{t}}\right)-1\right], \\
& I_{\mathrm{sh}}=\frac{V_{L}+R_{S} \cdot I_{L}}{R_{\mathrm{sh}}} \\
& V_{t}=\frac{k T}{q} .
\end{aligned}
$$

In equation (2), $V_{L}$ is output voltage, $I_{\text {sh }}$ represents diode reverse saturation current, and $n$ means diode ideality factor. Besides, $V_{t}$ can be written as equation (4) with $k$ being Boltzmann constant, $1.3806503 \times 10^{-23}(\mathrm{~J} / \mathrm{K}), q$ stands for electronic charge, and $T$ is the Kelvin temperature of cell. Thus, equation (1) can be rewritten as follows:

$$
I_{L}=I_{\mathrm{ph}}-I_{\mathrm{sd}} \cdot\left[\exp \left(\frac{V_{L}+R_{S} \cdot I_{L}}{n \cdot V_{t}}\right)-1\right]-\frac{V_{L}+R_{S} \cdot I_{L}}{R_{\mathrm{sh}}}
$$

with five unknown parameters $I_{\mathrm{ph}}, I_{\mathrm{sh}}, R_{S}, R_{\mathrm{sh}}$, and $n$. 


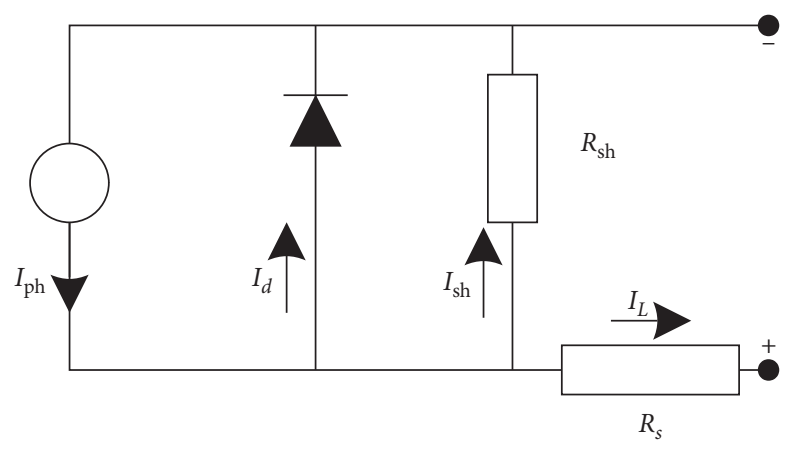

FIgURE 1: Equivalent circuit diagram of SDM.

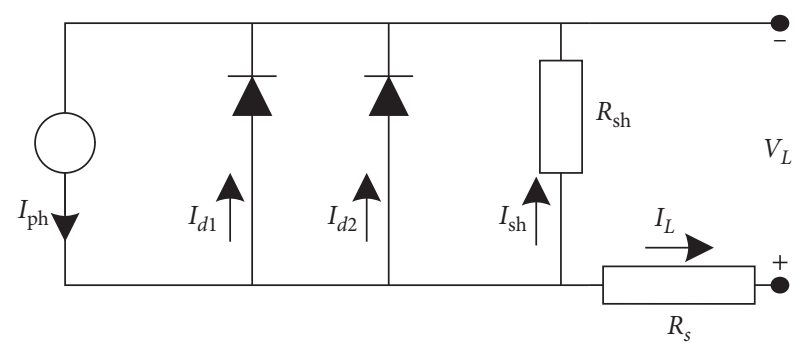

Figure 2: Equivalent circuit diagram of DDM.

2.1.2. DDM. Compared to SDM, DDM considers the effect of compound current loss, and its structure is displayed in Figure 2. As its name implies, DDM has two diodes in parallel with the current source. The output current of DDM is written mathematically as

$$
\begin{aligned}
I_{L} & =I_{\mathrm{ph}}-I_{\mathrm{sd}_{1}} \cdot\left[\exp \left(\frac{V_{L}+I_{L} \cdot R_{S}}{n_{1} \cdot V_{t}}\right)-1\right] \\
& -I_{\mathrm{sd}_{2}} \cdot\left[\exp \left(\frac{V_{L}+I_{L} \cdot R_{S}}{n_{2} \cdot V_{t}}\right)-1\right]-\frac{V_{L}+I_{L} \cdot R_{S}}{R_{\mathrm{sh}}},
\end{aligned}
$$

where $I_{\mathrm{sd}_{1}}$ and $I_{\mathrm{sd}_{2}}$ are, respectively, the diffusion current and saturation current and $n_{1}$ and $n_{2}$ are the ideality factors of two diodes. From equation (6), there are seven unknown parameters, $I_{\mathrm{ph}}, I_{\mathrm{sd}_{1}}, I_{\mathrm{sd}_{2}}, R_{S}, R_{\mathrm{sh}}, n_{1}$, and $n_{2}$, in DDM.

2.2. PV Module Model. PV module consists of multiple solar cells connected in series and in parallel, as shown in Figure 3. The SDM or DDM for PV modules can be represented by equation (7) or (8), respectively.

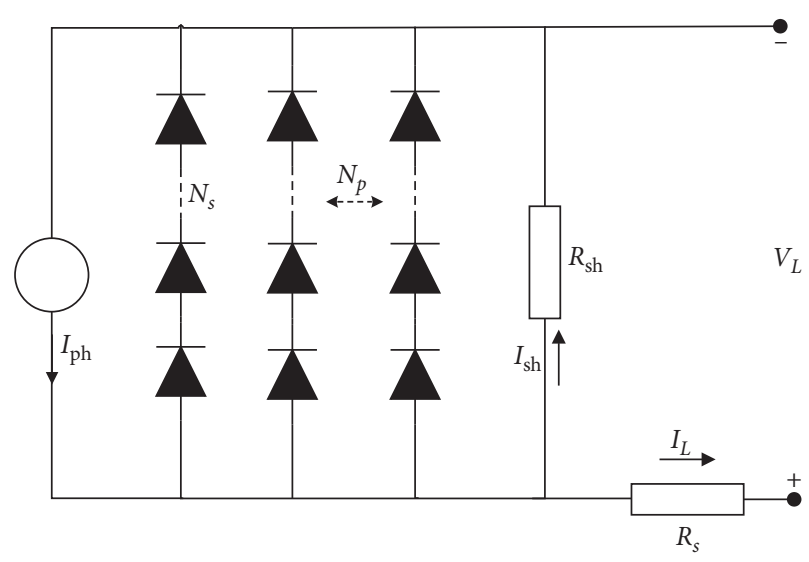

FIgURe 3: Equivalent circuit diagram of PV.

$$
\begin{aligned}
I_{L} & =I_{\mathrm{ph}} N_{p}-I_{\mathrm{sd}} N_{p}\left[\exp \left(\frac{V_{L}+\left(N_{s} R_{S} I_{L} / N_{p}\right)}{n N_{s} V_{t}}\right)-1\right] \\
& -\frac{V_{L}+\left(N_{s} R_{S} I_{L} / N_{p}\right)}{\left(N_{s} R_{\mathrm{sh}} / N_{p}\right)}
\end{aligned}
$$

$$
\begin{aligned}
I_{L} & =I_{\mathrm{ph}} N_{p}-I_{\mathrm{sd}_{1}} N_{p}\left[\exp \left(\frac{V_{L}+\left(N_{s} R_{S} I_{L} / N_{p}\right)}{n_{1} N_{s} V_{t}}\right)-1\right] \\
& -I_{\mathrm{sd}_{2}} N_{p}\left[\exp \left(\frac{V_{L}+\left(N_{s} R_{S} I_{L} / N_{p}\right)}{n_{2} N_{s} V_{t}}\right)-1\right] \\
& -\frac{V_{L}+\left(N_{s} R_{S} I_{L} / N_{p}\right)}{\left(N_{s} R_{\mathrm{sh}} / N_{p}\right)}
\end{aligned}
$$

where $N_{p}$ and $N_{s}$ are the number of solar cells in parallel and in series of the PV module, respectively. From the above two formulations, it is clear that the unknown parameters in the two PV module models are the same as those in SDM and in DDM.

2.3. Objective Function. The data of output voltage and output current are actually collected in the problem of parameter identification of PV cells and modules. In the identification process, all that needs to be done is to find the optimal set of values of unknown parameters which minimizes the error between the current calculated based on the simulation model and the actual measured one. Therefore, 
the root mean square of the error between these two current values is used as an objective function, as shown in equation (9), in this problem [58]. Thus, the problem studied in this paper can be equivalent to finding the optimal value of the objective function in

$$
\mathrm{RMSE}=\sqrt{\frac{\sum_{i=1}^{M}\left(I_{\text {simulated }}-I_{\text {measured }}\right)^{2}}{M}},
$$

where $I_{\text {simulated }}$ is the current estimated from the model, $I_{\text {mesured }}$ is the actual measured current, and $M$ is the quantity of current. Furthermore, the estimated current $I_{\text {simulated }}$ for each model can be obtained according to (5)-(8).

\section{Background of WOA}

3.1. The Basic WOA. WOA is an intelligent optimization algorithm implemented by simulating the foraging behavior of humpback whales. The algorithm divides the behavior of humpback whales into three parts: encircling prey, bubblenet attacking, and searching for prey.

3.1.1. Encircling Prey. In various solving problems, the optimal solution is not a priori known, so it is assumed that the current optimal solution is the target prey or is close to the prey in WOA. After the prey location is defined, individuals in humpback whale populations will attempt to update their locations in the direction of the prey by

$$
\overrightarrow{X(t+1)}=\overrightarrow{X_{\text {prey }}(t)}-\vec{A} \cdot \vec{D}
$$

where $t$ is the number of current iteration and $\overrightarrow{X_{\text {prey }}(t)}$ indicates the vector of current prey position. Both $\vec{A}$ and $\vec{D}$ are coefficient vectors given in the following equations:

$$
\begin{aligned}
& \vec{A}=2 \vec{r} \cdot \vec{a}-\vec{a}, \\
& \vec{D}=\left|\vec{C} \cdot \overrightarrow{X_{\text {prey }}(t)}-\overrightarrow{X(t)}\right| .
\end{aligned}
$$

In equation (11), $\vec{a}$ is the linearly decreasing parametric vector from 2 to 0 while $\vec{r}$ is the random vector within $[0,1]$. In equation (12), denotes the absolute value and $\vec{C}$ is the coefficient vector expressed as

$$
\vec{C}=2 \vec{r}
$$

3.1.2. Bubble-Net Attacking. Humpback whales swim simultaneously around their prey along a spiral path in a narrow circle, which is known as a bubble-net attack. Their behavior is simulated in WOA by both the contraction bracketing mechanism and the spiral model. In the optimization process, the selection probability of individuals in the population is the same for two ways. Besides, the specific mathematical model is written as follows:

$$
\begin{aligned}
& \overrightarrow{X(t+1)}=\left\{\overrightarrow{X_{\text {prey }}(t)}-\vec{A} \cdot \vec{D}, p<0.5, \vec{D} \cdot e^{b l} \cdot \cos (2 \pi l)+\overrightarrow{X_{\text {prey }}(t)}, p \geq 0.5,\right. \\
& \quad \overrightarrow{X(t+1)}=\overrightarrow{X_{\text {rand }}(t)}-\vec{A} \cdot \vec{D},
\end{aligned}
$$

where the parameter $b$ is the constant used to define the logarithmic spiral shape and $l$ is the random number within the interval $[-1,1]$ while $p$ is within $[0,1]$.

In equation (14), the whale chooses the contraction bracketing mechanism to update its position when $p<0.5$. Under this circumstance, the coefficient vector $\vec{A}$ varies with $\vec{a}$, and its variation is fixed within the interval $[-1,1]$. By selecting the contraction bracketing mechanism, the location of new individual will be defined anywhere between the original individual and the prey. When $p \geq 0.5$, the spiral model is chosen to update the position of new individual. In this event, the distance between the whale and the prey is firstly calculated, and $\vec{D}$ measures their distance expressed in equation (15). After that, a spiral equation is created between the position of the whale and the prey to simulate the spiral motion of a humpback whale.

$$
\vec{D}=\left|\overrightarrow{X_{\text {prey }}(t)}-\overrightarrow{X(t)}\right|
$$

3.1.3. Searching for Prey. In the stage of searching for prey, WOA updates the location of the population by the following equation: where $\overrightarrow{X_{\text {rand }}(t)}$ is a position vector of a random individual in the current population and $\vec{D}$ is calculated as follows:

$$
\vec{D}=\left|\vec{C} \cdot \overrightarrow{X_{\text {rand }}(t)}-\overrightarrow{X(t)}\right| .
$$

At this phase, humpback whales are randomly searched based on the positions of each other. Although the algorithm explores the best agent according to the change of $\vec{A}$, it is different from the contraction bracketing mechanism in which the module of $\vec{A}$ is greater than 1, which means that whales in the search are forced away from the prey location. In other words, WOA at this stage emphasizes global search.

3.2. Inferences from Literature and Motivation behind this Work. WOA has a strong global search capability because of its efficient location update method. Accordingly, its variants are applied in many fields, such as parameter evaluation [59], feature selection $[60,61]$, resource scheduling in cloud computing [62], and clustering [63]. The wide application of WOA in various fields directly shows that the algorithm is a developable tool in practical applications [64-67]. 
While the traditional WOA has strong global search capabilities, it also has weak local search capabilities due to its overreliance on stochastic convergence factors to convert exploration to exploitation. Therefore, it is an important task to help the basic WOA improve its local search capabilities. Because of its freedom of local derivative optimization, pattern search has been widely used in solving many optimization problems outside the scope of standard optimization techniques. Furthermore, in this work, pattern search has become a useful tool to improve the local search capabilities of WOA. Typically, in traditional swarm intelligence algorithms, the imbalance between exploration and exploitation is also an important factor affecting the performance of the algorithm. However, Levy flight is a nonGaussian random walk distribution model that has an indeterminate step length. The method is also highly stochastic and has a trajectory very similar to that of natural biological activity, which can help swarm intelligence algorithms to adjust the balance between exploration and exploitation. Therefore, the mechanism is considered in this study. Moreover, some improved WOAs have been designed and applied to the problem of photovoltaic model parameter evaluation. However, according to the law of no free lunch, it is still necessary to develop more optimizers to make the problem better solved.

\section{The Proposed Algorithm}

In this section, several mechanisms will be introduced in detail, and the framework of the proposed algorithm will be outlined.

\subsection{Basic Preliminaries of the Proposed Algorithm}

4.1.1. Levy Flight. Randomization plays a role in both exploration and exploitation as well as both diversification and intensification. In addition, the essence of randomization is a random walking, which is a random process that involves performing a series of consecutive random steps. Levy flight is one of these random processes. Moreover, Levy flight has been employed as an optimization technique to improve the performance of multiple metaheuristic algorithms [68-71]. Note that Levy flight is a random walking obeying the Levy distribution defined in the following equation:

$$
\operatorname{Levy}(s) \sim|s|^{-1-\beta}, \quad \beta \in(0,2),
$$

where $\beta$ is an exponential function that determines the shape of Levy distribution and $s$ is a random step, which can be expressed in the following form:

$$
s=\frac{\mu}{|\nu|^{(1 / \beta)}}
$$

with $\mu$ and $\nu$ being parameters subject to normal distribution, i.e., $\mu \sim N\left(0, \sigma_{\mu}^{2}\right)$ and $\nu \sim N\left(0, \sigma_{v}^{2}\right)$. The expressions for $\sigma_{\mu}$ and $\sigma_{\nu}$ are given as follows:

$$
\begin{aligned}
& \sigma_{\mu}=\left(\frac{\Gamma(1+\beta) \cdot \sin (\pi \beta / 2)}{\Gamma(1+\beta / 2) \cdot \beta \cdot 2^{(\beta-1 / 2)}}\right)^{(1 / \beta)}, \\
& \sigma_{\nu}=1,
\end{aligned}
$$

where $\Gamma$ stands for the gamma function.

4.1.2. Pattern Search. It is universally acknowledged that pattern search is a local derivative free optimization method which is ideal for solving many optimizations problems beyond the scope of standard optimization techniques.

The initial process of pattern search begins around a set of points called dots. Then, the grid is made by adding the nearest point to a set of scalar multiples of a vector called pattern. Besides, a point with better fitness is selected from the points in the grid as the initial point for the next iteration. The specific steps in the pattern search are as follows:

Step 1: determine the initial point $x_{0}$ and calculate its fitness $f\left(x_{0}\right)$.

Step 2: calculate other points $x_{i}$ around the initial point $x_{0}$ according to equation (22), while calculating their objective fitness $f\left(x_{i}\right)$. If there is one point better than $x_{0}$, perform step 3; otherwise, perform step 4 .

$$
x_{i}=x_{0}+v(j) \cdot L,
$$

where $v(j)$ indicates the pattern vector in which $j \in(1,2, \ldots, 2 d)$, with $d$ being the dimension of the problem to be solved, and $L$ signifies the search step.

Step 3: update $L$ based on equation (23), where $\delta>1$ and is used to expand the search space. Then execute step 5.

$$
L=\delta L .
$$

Step 4: update $L$ according to equation (24), where $\lambda<1$ and is used to narrow the search space. Then execute step 5.

$$
L=\lambda L
$$

Step 5: if termination condition is not met, continue with step 1; otherwise, stop the iteration and output the optimal point.

4.2. Framework of MWOA. We propose the modified whale optimization algorithm (MWOA) in this paper. The pseudocode of MWOA is shown as follows in Algorithm 1, and Figure 4 displays its flowchart. In MWOA, Levy flight is initially employed to improve the performance of the algorithm to avoid falling prematurely into a local optimum. In summary, MWOA first performs the basic strategy of the original whale optimization algorithm to update the population. Then, Levy flight is operated as follows. According to equation (25), the individual $V_{i}$ that has undergone Levy 
Initialize the whale's population $X$;

Calculate the fitness of each search agent;

Leader_pos = the best search agent;

while $(t<$ maximum number of iterations)

for each search agent

Update $a, A, C, l$ and $p$;

if $(p<0.5)$

if $(|A|<1)$

Update the position of the current search agent by equation (10);

else if $(|A| \geq 1)$

end if

Update the position of the current agent by equation (16);

else if $(p \geq 0.5)$

Update the position of the current search agent by equation (14);

end if

Carry on the Levy flight for each agents using equation (25);

end for

Using pattern search to the best agent Leader_pos;

Check if any search agent goes beyond the search space and amend it;

Calculate the fitness of each search agent;

Update Leader_pos if there is a better solution;

$t=t+1$

end while

return Leader_pos;

Algorithm 1: MWOA.

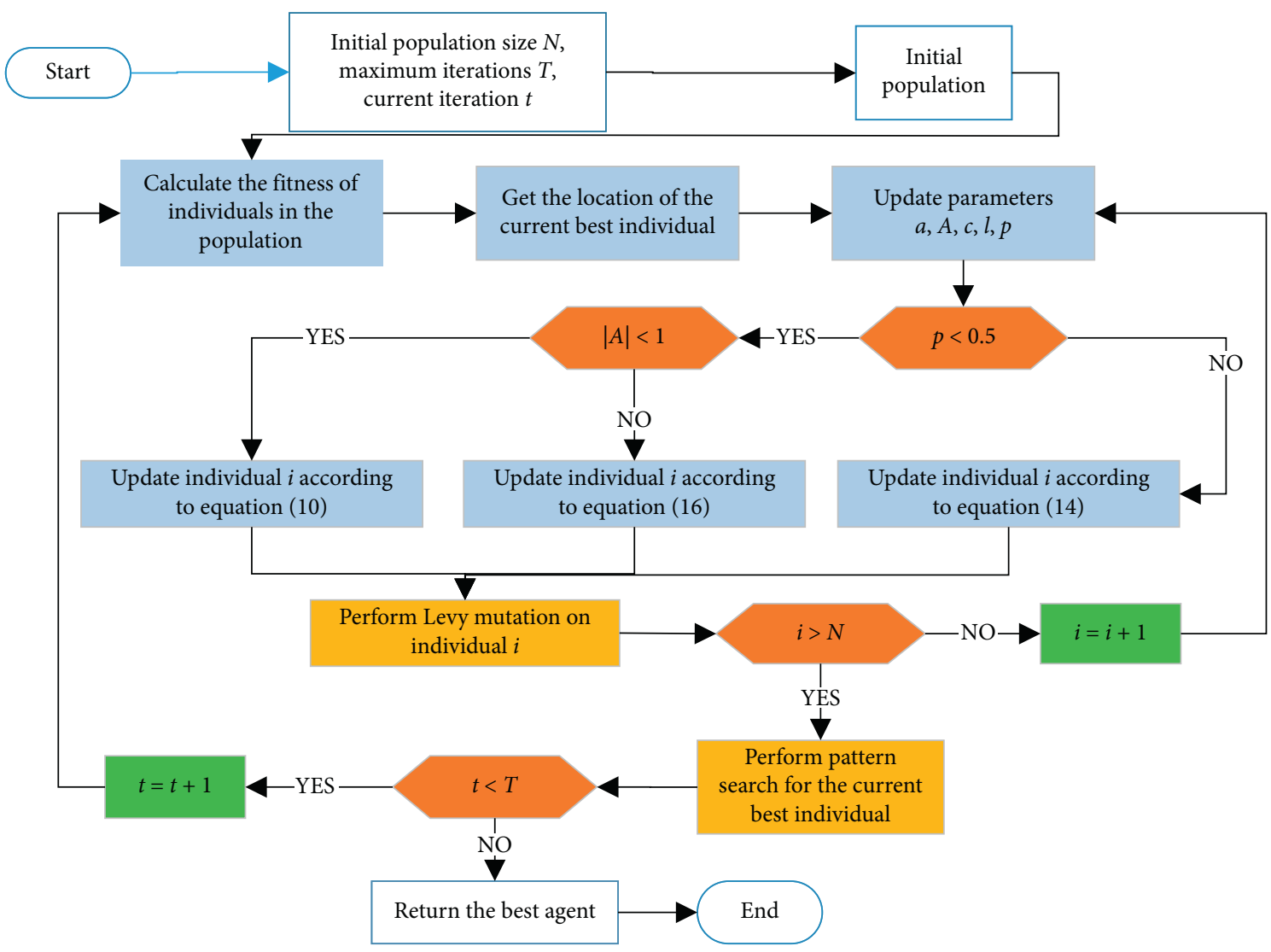

Figure 4: Flowchart of MWOA. 
variation is obtained and its fitness $f\left(V_{i}\right)$ is calculated concurrently. If $f\left(V_{i}\right)$ is better than $f\left(X_{i}\right)$, then $V_{i}$ will be substituted for $X_{i}$. In equation (25), Leader_pos means the current optimal solution and $\alpha$ is the constant coefficient with a value of 0.75 and $\beta=1.5$.

$$
V_{i}=X_{i}+\alpha \cdot \operatorname{Levy}(\beta) \oplus\left(X_{i}-\text { Leader_pos }\right) .
$$

After performing Levy flight on the entire population, the optimal solution in the current population is picked as the initial point of pattern search. A new solution, $X_{\mathrm{ps}}$, is found by pattern search. Then, compared with the current optimal solution, the better one is chosen. With respect to pattern search, the function patternsearch () in MATLAB is used directly. For the iteration termination condition of the pattern search, it is set to 0.1 times the maximum number of MWOA iterations, and the remaining parameters are default values.

4.3. Time Complexity Analysis. Before analyzing the time complexity of the proposed method, it is first divided into the following steps:

Step 1: initialization parameters: initialize the generation counter $t$, the number of maximum iterations $T$, the population size $N$, the dimensionality of the space $d$, and the boundary of the searching area $[l b, u b]$.

Step 2: initialization of random whale populations

Step 3: calculate the fitness of whales and choose the best agent

Step 4: update whales: update the whales in the population according to equations (10)-(17).

Step 5: Levy flight: get the variant $V$ by performing the Levy flight based on equation (25), and then update the population if the fitness of $V$ is better than the original one. Bring new whales back if there is someone outside the boundaries.

Step 6: pattern search: choose the current optimal Leader_pos to perform pattern search mechanism to generate the candidate position and bring it back if it is over the border. Then, update Leader_pos if the fitness of the candidate obtained previously is superior to that of Leader_pos.

Step 7: stop or continue the iterative process: repeat step 3 to step 8 until the exit condition is met, and then return Leader_pos.

Next, we proceed to the time complexity analysis. The primary time complexity of MWOA mainly relies on steps 3-7. Thus, the overall time complexity of MWOA $(O$ $($ MWOA $)=O$ (population initialization $)+G *(O$ (fitness evaluation $)+O$ (whales updating) $)+O$ (the Levy flight strategy) $+O$ (the pattern search mechanism)). Considering that the population has $n$ individuals, the time complexity of the initialization is $O(n * d)$, and computing the fitness of whales in population is $O(G *(n d))$. Besides, updating the location of the whales is $O(G *(6 n d+7 n))$. Thus, the time complexity of the original WOA is $O(n * d)+O$
$(G(n * \mathrm{~d}))+O(G *(6 n d+7 n))$. In addition, the time complexity of the Levy flight strategy is $O$ $(G *(n d+n d+4 n))$, while pattern search is $O$ $(G *(0.1 * G+6))$. Therefore, the time complexity of MWOA is approximately equal to $O(n d)+O$ $(G *(9 n d+11 n+0.1 G+6))$. Note that both the proposed MWOA and the classical WOA have a complexity of $O(n d)$ based on the large $O$ notation.

\section{Experimental Results and Analysis}

To verify the effectiveness of MWOA, parameter identification of solar cells and PV modules is carried out for the two datasets provided in the literature [72]. These two datasets relate to RTC France cell and Photowatt-PWP 201 PV module, and they have been widely used to validate the performance of the new methods $[3,73,74]$. The dataset of RTC France contains 26 pairs of current and voltage values measured under standard test conditions $\left(33^{\circ} \mathrm{C}, 1000 \mathrm{~W} / \mathrm{m}^{2}\right.$ light intensity) while that of Photowatt-PWP 201 consisting of 36 polysilicon cells in series includes 25 pairs of data measured under standard test conditions $\left(45^{\circ} \mathrm{C}, 1000 \mathrm{~W} / \mathrm{m}^{2}\right.$ light intensity).

In this part, MWOA was implemented by MATLAB R2014a and was conducted in 30 runs independently on each model. Each experiment was terminated by reaching the maximum number $(10,000)$ of iterations. The range of parameters to be identified for SDM, DDM, and PV module is reported in Table 1.

To further check the validity and reliability of MWOA, it was applied to estimate parameters of the two different types of PV modules: thin-film PV module ST40 and monocrystalline silicon PV module SM55. The experimental data are obtained from their product data book, including data under different light intensity and temperatures. In this part of experiment, the maximum iterations and the population size were set to 5000 and 30 , respectively.

5.1. Results of RTC France Solar Cell. In this part, the experimental results of MWOA on single diode and double diode solar cells and photovoltaic modules will be given in detail. Among them, because some competing algorithms have relatively good performance on a certain model, it may perform poorly on other models. Therefore, the selected competition algorithm on the three models may be different. However, to ensure fairness, the settings of each algorithm are consistent on the same model.

5.1.1. Results of SDM. Table 2 lists the results including the values of five parameters in SDM and RMSE based on MWOA along with the other seven algorithms for comparison, which are CPSO [75], LMSA [76], EHHO [77], ABSO [3], GOTLBO [78], LWOA [49], and CWOA [79]. Additionally, Figure 5 demonstrates the I-V characteristic (I-VC) and the P-V characteristic (P-VC) of the best SDM obtained based on MWOA. To further reveal the quality of the results, Table 3 lists the absolute errors (IAEs) and the relative errors (REs) between the simulated and the 
TABLE 1: Bounds of parameters for three different models.

\begin{tabular}{|c|c|c|c|c|}
\hline \multirow{2}{*}{ Parameters } & \multicolumn{2}{|c|}{$\begin{array}{l}\text { Single diode/double } \\
\text { diode }\end{array}$} & \multicolumn{2}{|c|}{ PV module } \\
\hline & $\begin{array}{l}\text { Lower } \\
\text { bound }\end{array}$ & $\begin{array}{l}\text { Upper } \\
\text { bound }\end{array}$ & $\begin{array}{l}\text { Lower } \\
\text { bound }\end{array}$ & $\begin{array}{l}\text { Upper } \\
\text { bound }\end{array}$ \\
\hline$I_{\mathrm{ph}}(\mathrm{A})$ & 0 & 1 & 0 & 2 \\
\hline$I_{\mathrm{sd}}(\mu \mathrm{A})$ & 0 & 1 & 0 & 50 \\
\hline$R_{s}(\Omega)$ & 0 & 0.5 & 0 & 2 \\
\hline$R_{\mathrm{sh}}(\Omega)$ & 0 & 100 & 0 & 2000 \\
\hline$N$ & 1 & 2 & 1 & 50 \\
\hline$I_{\mathrm{sd}_{1}}(\mu \mathrm{A})$ & 0 & 1 & 0 & 50 \\
\hline$I_{\mathrm{sd}_{2}}(\mu \mathrm{A})$ & 0 & 1 & 0 & 50 \\
\hline$n_{1}$ & 1 & 2 & 1 & 50 \\
\hline$n_{2}$ & 1 & 2 & 1 & 50 \\
\hline
\end{tabular}

experimental data, while Figure 6 displays the IAE and the $\mathrm{RE}$ between these data with their definitions given in equations (26) and (27), respectively. Besides, IAE and RE can more intuitively help readers observe the error between the actual measured data and the data obtained by the simulation experiment at each measurement point, thereby further reflecting the performance of the proposed algorithm.

$$
\begin{gathered}
\mathrm{IAE}=\left|I_{\text {measured }}-I_{\text {simulated }}\right|, \\
\mathrm{RE}=\frac{\left|I_{\text {measured }}-I_{\text {simulated }}\right|}{I_{\text {measured }}} .
\end{gathered}
$$

We can see from Table 3 that, for SDM, MWOA not only performs better than other competing algorithms, but also has more satisfactory optimization accuracy compared to other improved WOA methods. As shown in Figure 6, I-VC and P-VC of the best SDM obtained by the method proposed in this paper are in good agreement with the experimental data over all measured points. Moreover, the IAEs of all simulated currents are less than 8.25668E-05, and the corresponding REs are within the interval $[-1.9962 e-02$, $2.1517 e-02]$. Above all, we conclude that the actual behavior of solar cells can be accurately described by SDM which is estimable by MOWA.

5.1.2. Results of DDM. For DDM of RTC France, the values of parameters and RMSE obtained based on the seven different algorithms including MWOA are reported in Table 4: SA [80], HS [81], GGHS [81], IGHS [81], LWOA, CWOA, and MWOA. Besides, Figure 7 depicts the comparison of I-VC and P-VC of the estimated DDM based on MWOA with the experimental data. Furthermore, the IAE and the RE of simulated currents are shown in Figure 8 while the specific values of simulated data are provided in Table 5 .

It can be evidently seen from the figures and tables mentioned above that MWOA still performs well for DDM although it is slightly inferior to some algorithms. From the perspective of fitness, RMSE obtained by MWOA is worse than $\mathrm{ABC}$ and ICHS, only ranking the third place. However, MWOA is much better than the remaining algorithms. I-VC and $\mathrm{P}-\mathrm{VC}$ of the estimated model are in good agreement with the experimental data. Moreover, all the IAE of simulated currents are less than $2.524 E-02$, and all RE are within the interval $[-2.0108 E-02,1.3199 E-01]$. All of these results show that it is possible to identify the unknown parameters of DDM with high precision based on MWOA.

5.2. Results of Photowatt-PWP 201 Module. We need to identify five parameters in PV module model, and the results based on the eight algorithms are given in Table 6: Newton, CPSO, EHHO, CARO, LWOA, CWOA MWOA, and the method in [82]. As we can see in the table, MWOA produces the smallest RMSE $(2.42508 E-03)$ among all the algorithms, while CARO and LWOA are, respectively, the second and the third best in terms of RMSE. Moreover, the accuracy of MOWA is much higher than that of the other algorithms, indicating that the improved approach is effective in improving the performance of WOA in the parameter recognition of PV modules. Figure 9 shows the comparison of I-VC and P-VC between the best model constructed by MWOA and the experimental data. From Figure 9, we can see that the fit between these two is high. IAE and RE of the simulated currents are also shown in Figure 10. In the meantime, specific data including currents, powers, and their related IAE and RE are available in Table 7. We can see from the table that all the IAE of the simulated currents are less than $4.84 E-03$, while all $\mathrm{RE}$ are within the interval $[-3.973 E-02,5.0336 E-02]$. Therefore, MWOA is satisfactory for the parameter discrimination of PV module model.

Figure 11 presents the convergence curve of MWOA for three models, which reveals the performance in 30 runs. It is obvious that the proposed method converges faster and better than the original algorithm for parameter identification in SDM, DDM, and PV module. Moreover, compared to the newly published $\mathrm{EHHO}$ and another improved WOA called CWOA, MWOA always has the fastest convergence speed and the most prominent accuracy.

Based on the performance of the above MWOA on the three models, it can be found that, in terms of optimization accuracy, MWOA not only is better than other competitive algorithms, but also has a competitive advantage compared to other improved WOAs. Accordingly, there is a reason to say that MWOA is a promising tool in the field of evaluating unknown parameters of photovoltaic models.

5.3. Discussion. It can be known from the above that MWOA introduces two strategies, Levy flight and pattern search. In order to further prove the contribution of these two mechanisms to the proposed algorithm, the maximum (max), minimum (min), mean value (mean), and standard deviation (std) of RMSE are introduced. Table 8 shows the comparison results of MWOA with its variants involving only one of these mechanisms. Among them, the method that only introduces Levy flight is recorded as LWOA, and the method that only involves pattern search is recorded as PSWOA. From Table 8, whether it is the max, min, or mean, MWOA always has the best performance in the three experimental models, which demonstrates that MWOA has the most satisfactory optimization accuracy. What is more, 
TABLE 2: Identification results of SDM based on seven algorithms.

\begin{tabular}{lcccccccc}
\hline Item & CPSO & LMSA & EHHO & ABSO & GOTLBO & LWOA & CWOA & MWOA \\
\hline$I_{\text {ph }}(\mathrm{A})$ & 0.7607 & 0.76078 & 0.7611 & 0.7608 & 0.760780 & 0.7602 & 0.7605 \\
$I_{\text {sd }}(\mu \mathrm{A})$ & 0.4 & 0.31849 & 0.3613 & 0.30623 & 0.331552 & 0.4607 & 0.5047 & 3.23 \\
$R_{s}(\Omega)$ & 0.0354 & 0.03643 & 0.03583 & 0.03659 & 0.036265 & 0.0350 & 0.0341 \\
$R_{\text {sh }}(\Omega)$ & 59.012 & 53.32644 & 53.752 & 52.2903 & 54.11543 & 75.4619 & 51.4778 & 53.76689 \\
$n$ & 1.5033 & 1.47976 & 1.4814 & 1.47878 & 1.483820 & 1.5177 & 1.52783 & 1.48127 \\
RMSE & $1.3900 E-03$ & $9.8640 E-04$ & $1.0484 E-03$ & $9.9124 E-04$ & $9.8744 E-04$ & $1.2352 E-03$ & $1.5792 E-03$ & $\mathbf{9 . 8 6 0 2 E - 0 4}$ \\
\hline
\end{tabular}

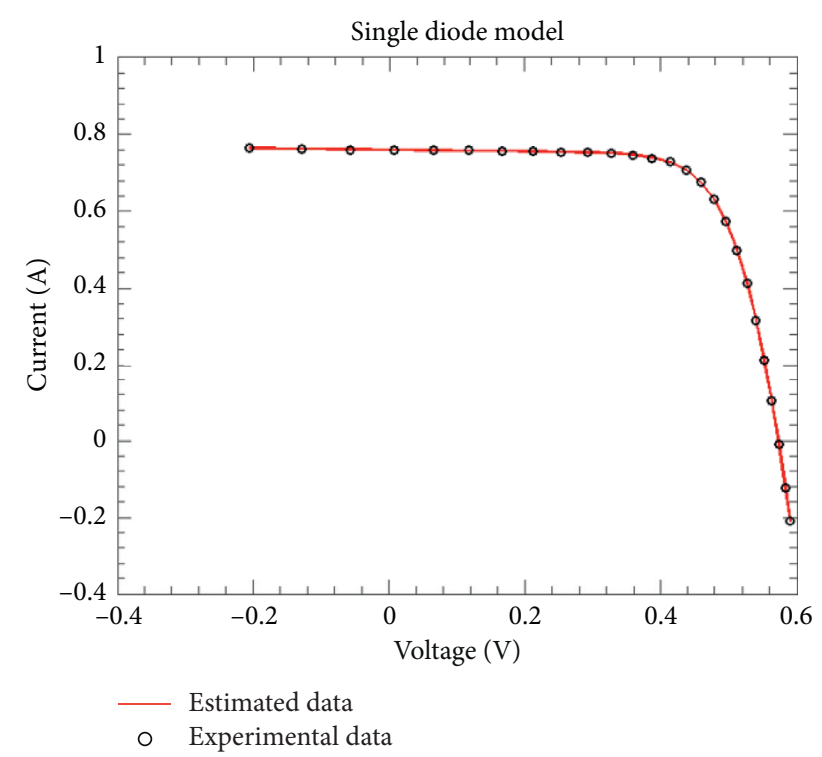

(a)

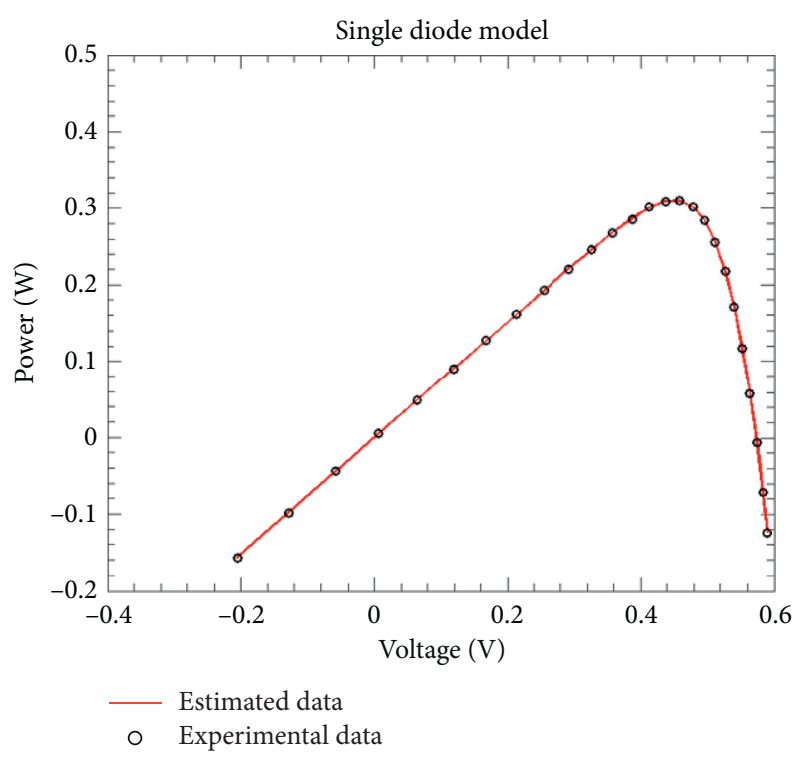

(b)

FIGURE 5: I-VC (a) and P-VC (b) of the best SDM obtained based on MWOA.

TABLE 3: Simulated data and associated IAE obtained by MWAO for SDM.

\begin{tabular}{|c|c|c|c|c|c|c|}
\hline \multirow{2}{*}{ Item } & \multicolumn{2}{|c|}{ Measured data } & \multicolumn{2}{|c|}{ Simulated current data } & \multicolumn{2}{|c|}{ Simulated power data } \\
\hline & $V(V)$ & $I(A)$ & $I_{\text {sim }}(A)$ & $\operatorname{IAE}_{I}(A)$ & $P_{\text {sim }}(W)$ & $\operatorname{IAE}_{P}(W)$ \\
\hline 1 & -0.2057 & 0.7640 & 0.764082567 & $8.25668 E-05$ & -0.15717178 & $1.69840 E-05$ \\
\hline 2 & -0.1291 & 0.7620 & 0.762659230 & 0.000659230 & -0.09845930 & $8.51067 E-05$ \\
\hline 3 & -0.0588 & 0.7605 & 0.761352627 & 0.000852627 & -0.04476753 & $5.01345 E-05$ \\
\hline 4 & 0.0057 & 0.7605 & 0.760152391 & 0.000347609 & 0.004332869 & $1.98137 E-06$ \\
\hline 5 & 0.0646 & 0.7600 & 0.759054593 & 0.000945407 & 0.049034927 & $6.10733 E-05$ \\
\hline 6 & 0.1185 & 0.7590 & 0.758042625 & 0.000957375 & 0.089828051 & 0.000113449 \\
\hline 7 & 0.1678 & 0.7570 & 0.757092741 & $9.27410 E-05$ & 0.127040162 & $1.55619 E-05$ \\
\hline 8 & 0.2132 & 0.7570 & 0.756143167 & 0.000856833 & 0.161209723 & 0.000182677 \\
\hline 9 & 0.2545 & 0.7555 & 0.755089260 & 0.000410740 & 0.192170217 & 0.000104533 \\
\hline 10 & 0.2924 & 0.7540 & 0.753666678 & 0.000333322 & 0.220372137 & $9.74633 E-05$ \\
\hline 11 & 0.3269 & 0.7505 & 0.751393925 & 0.000893925 & 0.245630674 & 0.000292224 \\
\hline 12 & 0.3585 & 0.7465 & 0.747356624 & 0.000856624 & 0.267927350 & 0.000307100 \\
\hline 13 & 0.3873 & 0.7385 & 0.740119380 & 0.001619380 & 0.286648236 & 0.000627186 \\
\hline 14 & 0.4137 & 0.7280 & 0.727383310 & 0.000616690 & 0.300918475 & 0.000255125 \\
\hline 15 & 0.4373 & 0.7065 & 0.706972335 & 0.000472335 & 0.309159002 & 0.000206552 \\
\hline 16 & 0.4590 & 0.6755 & 0.675278304 & 0.000221696 & 0.309952741 & 0.000101759 \\
\hline 17 & 0.4784 & 0.6320 & 0.630755139 & 0.001244861 & 0.301753258 & 0.000595542 \\
\hline 18 & 0.4960 & 0.5730 & 0.571924465 & 0.001075535 & 0.283674535 & 0.000533465 \\
\hline 19 & 0.5119 & 0.4990 & 0.499603044 & 0.000603044 & 0.255746798 & 0.000308698 \\
\hline 20 & 0.5265 & 0.4130 & 0.413645441 & 0.000645441 & 0.217784324 & 0.000339824 \\
\hline 21 & 0.5398 & 0.3165 & 0.317507878 & 0.001007878 & 0.171390753 & 0.000544053 \\
\hline 22 & 0.5521 & 0.2120 & 0.212154111 & 0.000154111 & 0.117130285 & $8.50846 E-05$ \\
\hline 23 & 0.5633 & 0.1035 & 0.102251839 & 0.001248161 & 0.057598461 & 0.000703089 \\
\hline 24 & 0.5736 & -0.0100 & -0.00871617 & 0.001283834 & -0.00499959 & 0.000736407 \\
\hline 25 & 0.5833 & -0.1230 & -0.12550541 & 0.002505411 & -0.07320731 & 0.001461406 \\
\hline 26 & 0.5900 & -0.2100 & -0.20847066 & 0.001529341 & -0.12299877 & 0.000902311 \\
\hline Sum of IAE & NA & NA & NA & 0.021516718 & NA & 0.008728789 \\
\hline
\end{tabular}




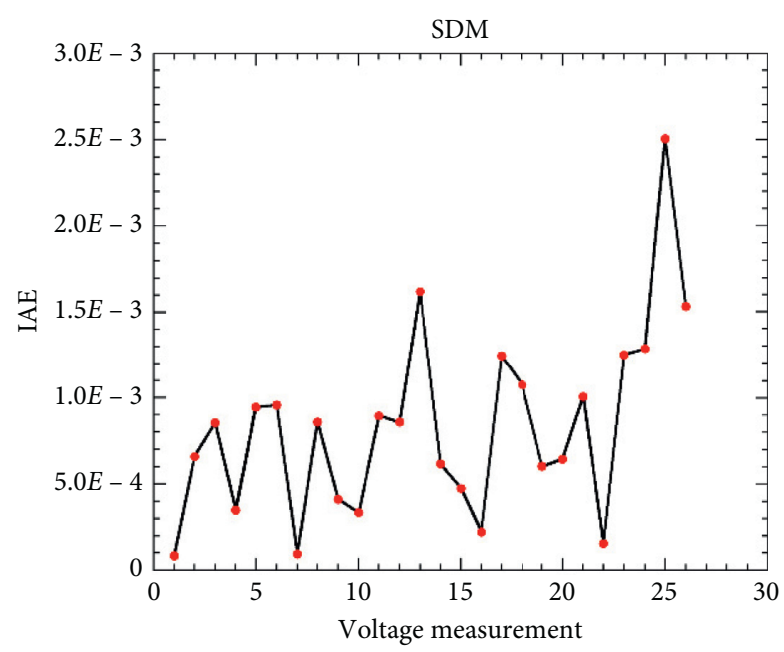

(a)

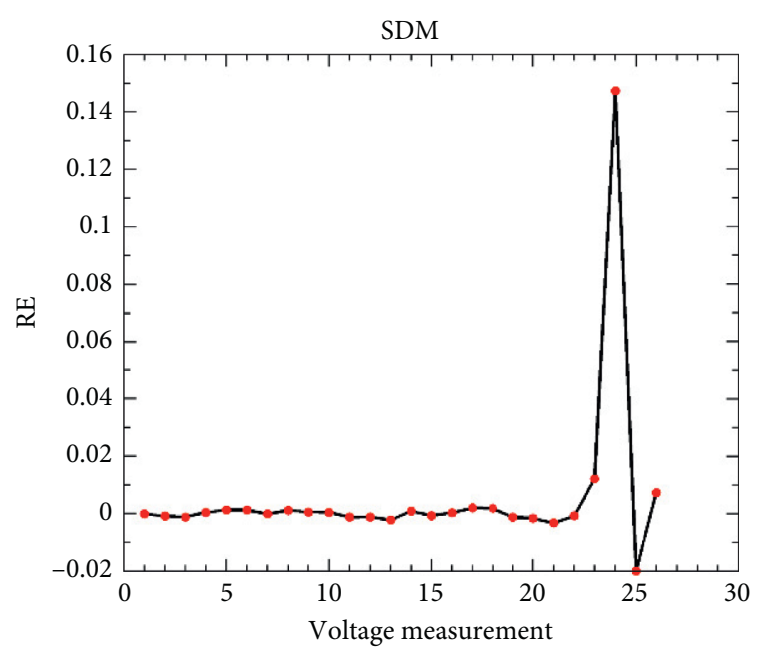

(b)

FIgURE 6: IAE (a) and RE (b) between the simulated data by SDM and the experimental data.

TABLE 4: Identification results of DDM based on seven algorithms.

\begin{tabular}{lcccccccc}
\hline Item & SA & HS & GGHS & IGHS & EHHO & LWOA & CWOA & MWOA \\
\hline$I_{\text {ph }}(\mathrm{A})$ & 0.7623 & 0.76176 & 0.7606 & 0.7608 & 0.7603 & 0.7608 & 0.7598 & 0.7608 \\
$I_{\text {sd }_{1}}(\mu \mathrm{A})$ & 0.4767 & 0.1255 & 0.3701 & 0.9731 & 0.0602 & 0.1667 & 0.2415 \\
$I_{\text {sd }_{2}}(\mu \mathrm{A})$ & 0.0100 & 0.2547 & 0.1350 & 0.1679 & 0.2273 & 0.0361 & 0.6000 \\
$R_{s}(\Omega)$ & 0.0345 & 0.03545 & 0.03562 & 0.0369 & 0.0349 & 55.2366 & 1.4565 & 3.6500 \\
$R_{\text {sh }}(\Omega)$ & 43.1034 & 46.8270 & 62.7899 & 53.8368 & 79.1512 & 1.6086 & 1.9899 & 53.3477 \\
$n_{1}$ & 1.5172 & 1.49439 & 1.49638 & 1.9213 & 1.95621 & 0.2323 & 0.0367 & 1.4638 \\
$n_{2}$ & 2.0000 & 1.4999 & 1.9300 & 1.4281 & 1.5025 & 1.4658 & 55.2016 \\
RMSE & $1.664 E-02$ & $1.26 E-03$ & $1.07 E-03$ & $9.8635 E-04$ & $1.312 E-04$ & $1.000 E-03$ & $1.789 E-03$ & $\mathbf{9 . 8 6 9 4 E}-\mathbf{0 4}$ \\
\hline
\end{tabular}

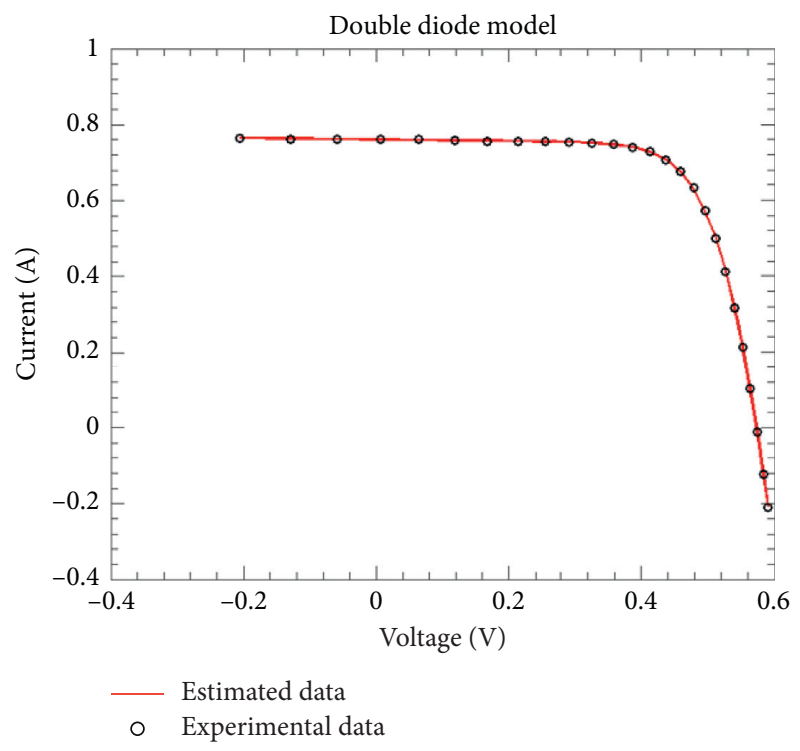

(a)

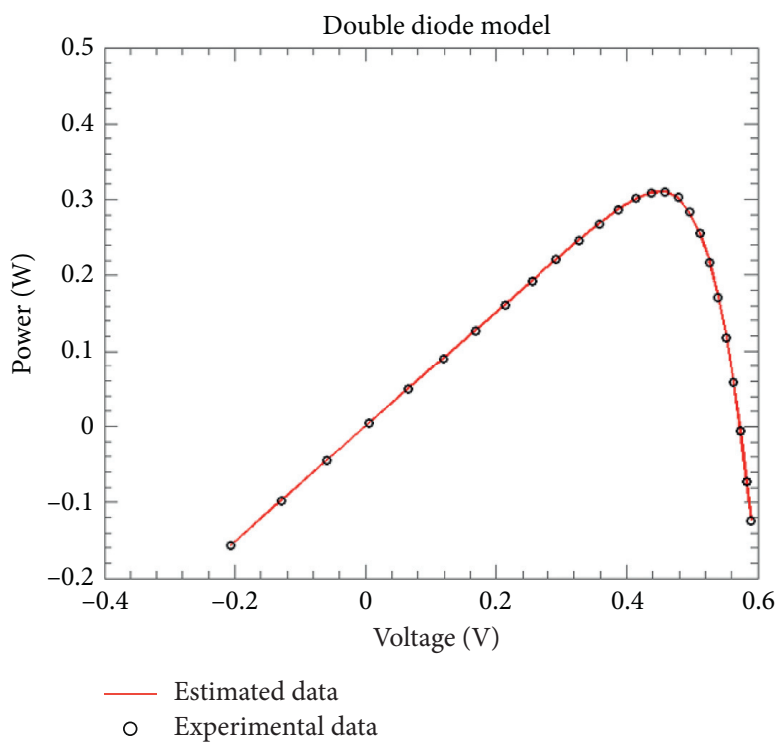

(b)

Figure 7: I-VC (a) and P-VC (b) of the best DDM obtained based on MWOA. 


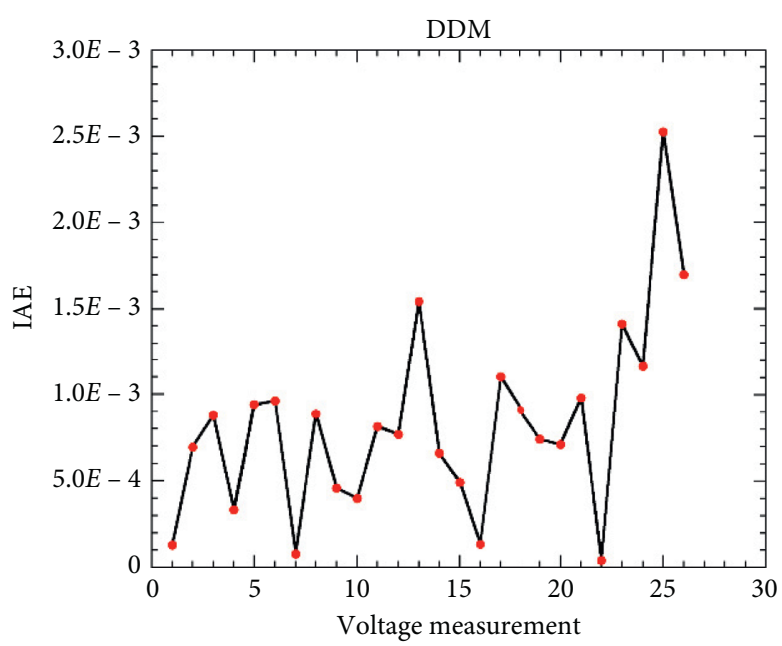

(a)

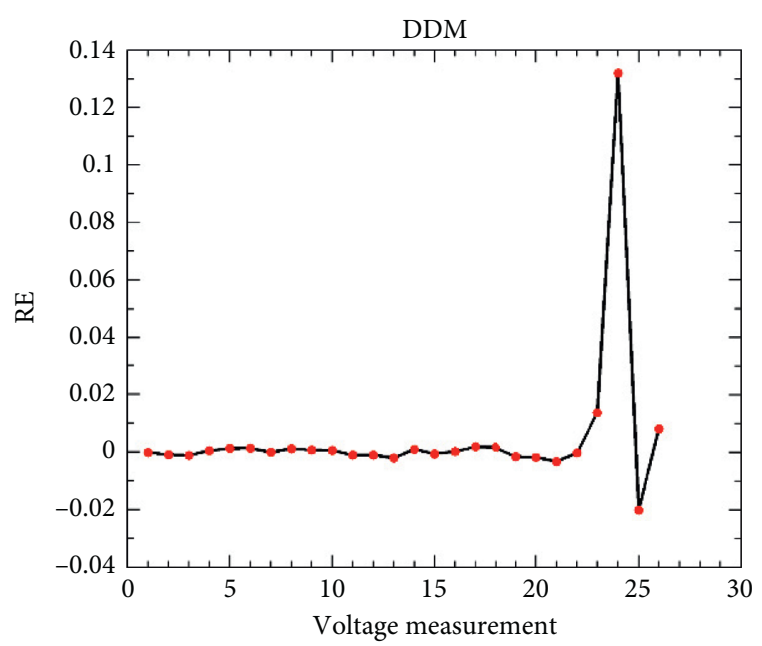

(b)

Figure 8: IAE (a) and RE (b) between the simulated data by DDM and the experimental data.

TABLE 5: Simulated data and associated IAE obtained by MWAO on SDM.

\begin{tabular}{|c|c|c|c|c|c|c|}
\hline \multirow{2}{*}{ Item } & \multicolumn{2}{|c|}{ Measured data } & \multicolumn{2}{|c|}{ Simulated current data } & \multicolumn{2}{|c|}{ Simulated power data } \\
\hline & $V(V)$ & $I(A)$ & $I_{\text {sim }}(A)$ & $\operatorname{IAE}_{I}(A)$ & $P_{\text {sim }}(W)$ & $\operatorname{IAE}_{P}(A)$ \\
\hline 1 & -0.2057 & 0.7640 & 0.764128657 & 0.000128657 & -0.15718127 & $2.64647 E-05$ \\
\hline 2 & -0.1291 & 0.7620 & 0.762694119 & 0.000694119 & -0.09846381 & $8.96107 E-05$ \\
\hline 3 & -0.0588 & 0.7605 & 0.761377154 & 0.000877154 & -0.04476898 & $5.15766 E-05$ \\
\hline 4 & 0.0057 & 0.7605 & 0.760167171 & 0.000332829 & 0.004332953 & $1.89713 E-06$ \\
\hline 5 & 0.0646 & 0.7600 & 0.759059926 & 0.000940074 & 0.049035271 & $6.07288 E-05$ \\
\hline 6 & 0.1185 & 0.7590 & 0.758038137 & 0.000961863 & 0.089827519 & 0.000113981 \\
\hline 7 & 0.1678 & 0.7570 & 0.757077118 & $7.71183 E-05$ & 0.127037540 & $1.29405 E-05$ \\
\hline 8 & 0.2132 & 0.7570 & 0.756113872 & 0.000886128 & 0.161203478 & 0.000188922 \\
\hline 9 & 0.2545 & 0.7555 & 0.755043390 & 0.000456610 & 0.192158543 & 0.000116207 \\
\hline 10 & 0.2924 & 0.7540 & 0.753602153 & 0.000397847 & 0.220353270 & 0.000116330 \\
\hline 11 & 0.3269 & 0.7505 & 0.751312692 & 0.000812692 & 0.245604119 & 0.000265669 \\
\hline 12 & 0.3585 & 0.7465 & 0.747267653 & 0.000767653 & 0.267895454 & 0.000275204 \\
\hline 13 & 0.3873 & 0.7385 & 0.740041090 & 0.001541090 & 0.286617914 & 0.000596864 \\
\hline 14 & 0.4137 & 0.7280 & 0.727341210 & 0.000658790 & 0.300901058 & 0.000272542 \\
\hline 15 & 0.4373 & 0.7065 & 0.706990350 & 0.000490350 & 0.309166880 & 0.000214430 \\
\hline 16 & 0.4590 & 0.6755 & 0.675367421 & 0.000132579 & 0.309993646 & $6.08538 E-05$ \\
\hline 17 & 0.4784 & 0.6320 & 0.630900439 & 0.001099561 & 0.301822770 & 0.000526030 \\
\hline 18 & 0.4960 & 0.5730 & 0.572089519 & 0.000910481 & 0.283756402 & 0.000451598 \\
\hline 19 & 0.5119 & 0.4990 & 0.499742182 & 0.000742182 & 0.255818023 & 0.000379923 \\
\hline 20 & 0.5265 & 0.4130 & 0.413712372 & 0.000712372 & 0.217819564 & 0.000375064 \\
\hline 21 & 0.5398 & 0.3165 & 0.317482559 & 0.000982559 & 0.171377085 & 0.000530385 \\
\hline 22 & 0.5521 & 0.2120 & 0.212040732 & $4.07323 E-05$ & 0.117067688 & $2.24883 E-05$ \\
\hline 23 & 0.5633 & 0.1035 & 0.102089898 & 0.001410102 & 0.057507240 & 0.000794310 \\
\hline 24 & 0.5736 & -0.0100 & -0.00883403 & 0.001165972 & -0.00506720 & 0.000668801 \\
\hline 25 & 0.5833 & -0.1230 & -0.12552400 & 0.002523998 & -0.07321815 & 0.001472248 \\
\hline 26 & 0.5900 & -0.2100 & -0.20830259 & 0.001697414 & -0.12289853 & 0.001001474 \\
\hline Sum of IAE & NA & NA & NA & 0.021441 & NA & 0.008687 \\
\hline
\end{tabular}

the smallest std indicates that MWOA has the best robustness on the three models. In conclusion, there is no way to achieve the expected results with a single mechanism, but the combination of the two can have better performance.

Besides, time Cost records the average CPU time of three versions of WOA after running independently for 30 times on a PC with main frequency of $3.4 \mathrm{GHZ}$, a memory of $8 \mathrm{~GB}$, and an operating system of Win 10 with MATLAB R2016a implementation. It can be seen that although the pattern search algorithm has strong local search capabilities, its introduction has significantly increased the computational cost. Nevertheless, it is worthwhile to spend 
TABLE 6: Identification results of PV module model based on eight algorithms.

\begin{tabular}{|c|c|c|c|c|c|c|c|c|}
\hline Item & Newton [72] & CPSO & EHHO & Method in [82] & CARO [83] & LWOA & CWOA & MWOA \\
\hline$I_{\mathrm{ph}}(\mathrm{A})$ & 1.0318 & 1.0286 & 1.0302 & 1.0310 & 1.03185 & 1.0293 & 1.0272 & 1.030529 \\
\hline$I_{\mathrm{sd}}(\mu \mathrm{A})$ & 3.2875 & 8.3010 & 3.6469 & 3.8236 & 3.28401 & 3.6916 & 4.2334 & 3.4744947 \\
\hline$R_{s}(\Omega)$ & 1.2057 & 1.0755 & 1.1964 & 1.0920 & 1.20556 & 1.1985 & 1.1879 & 1.201498 \\
\hline$R_{\mathrm{sh}}(\Omega)$ & 555.5556 & 1850.100 & 833.333 & 689.660 & 841.321 & 11198.783 & 1923.9615 & 979.3934 \\
\hline$n$ & 48.4500 & 52.2430 & 48.8202 & 48.9300 & 48.4036 & 48.8626 & 49.390 & 48.6343 \\
\hline RMSE & $7.8050 E-01$ & $3.5000 E-03$ & $2.429 E-03$ & $1.02 E-01$ & $2.427 E-03$ & $2.4529 E-03$ & $2.5962 E-03$ & $2.42508 \mathrm{E}-03$ \\
\hline
\end{tabular}

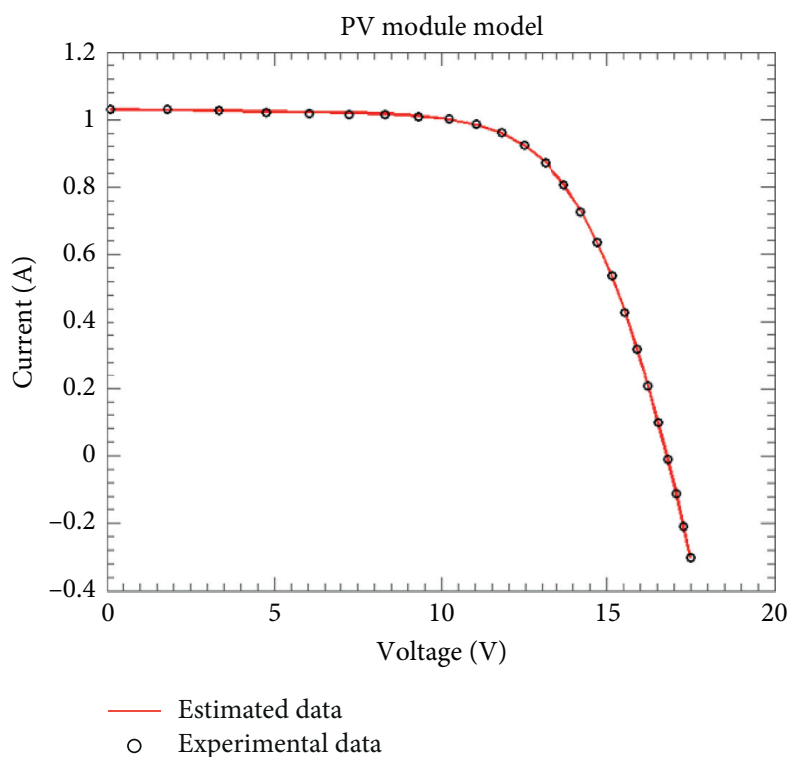

(a)

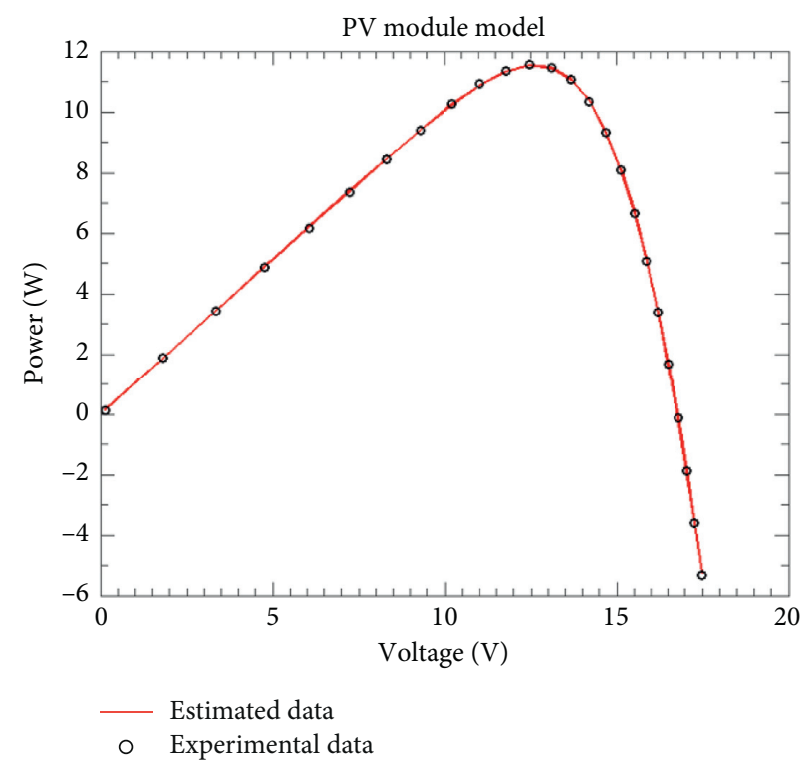

(b)

FIgURE 9: I-VC (a) and P-VC (b) of the best PV module model obtained based on MWOA.

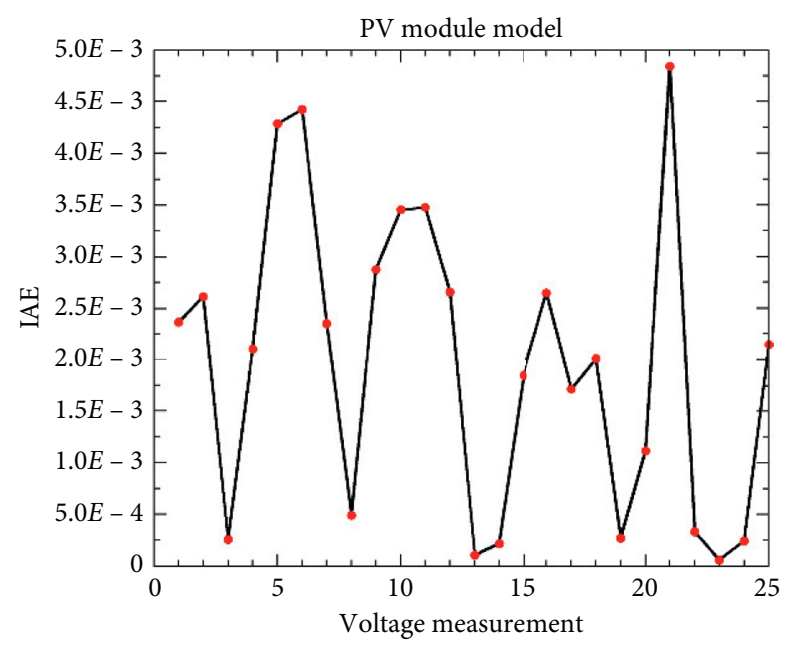

(a)

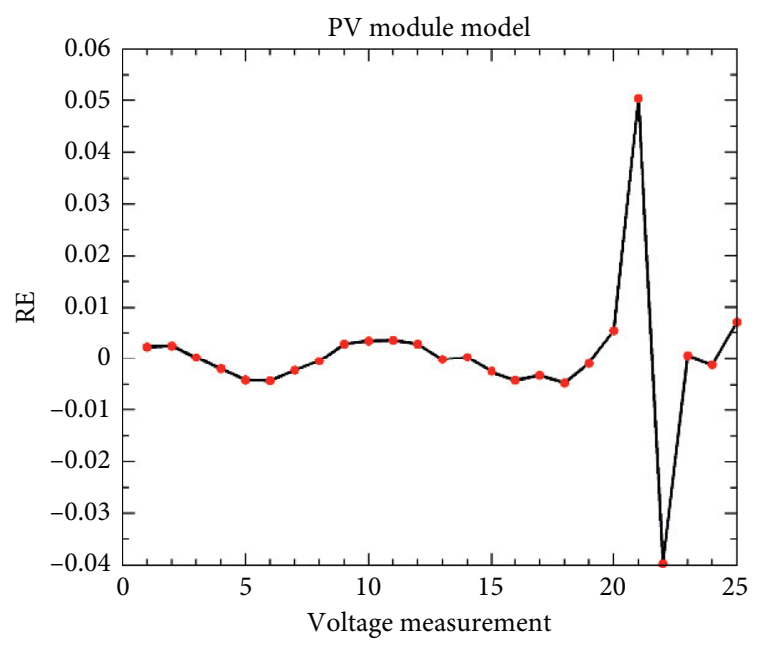

(b)

Figure 10: IAE (a) and RE (b) between the simulated data by PV module model and the experimental data.

a certain amount of time as a cost to improve accuracy. However, for large-scale problems, computing time will be an important issue worthy of attention. Therefore, reducing the computational cost without changing the accuracy is still a problem we need to pay attention to in the future. 
TABLE 7: Simulated data and associated IAE obtained by MWAO on PV module model.

\begin{tabular}{|c|c|c|c|c|c|c|}
\hline \multirow{2}{*}{ Item } & \multicolumn{2}{|c|}{ Measured data } & \multicolumn{2}{|c|}{ Simulated current data } & \multicolumn{2}{|c|}{ Simulated power data } \\
\hline & $V(V)$ & $I(A)$ & $I_{\text {sim }}(A)$ & $\operatorname{IAE}_{I}(A)$ & $P_{\text {sim }}(W)$ & $\operatorname{IAE}_{P}(A)$ \\
\hline 1 & 0.1248 & 1.0315 & 1.029129620 & 0.002370380 & 0.128435377 & 0.000295823 \\
\hline 2 & 1.8093 & 1.0300 & 1.027387041 & 0.002612959 & 1.858851373 & 0.004727627 \\
\hline 3 & 3.3511 & 1.0260 & 1.025743729 & 0.000256271 & 3.437369810 & 0.000858790 \\
\hline 4 & 4.7622 & 1.0220 & 1.024105540 & 0.002105540 & 4.876995401 & 0.010027001 \\
\hline 5 & 6.0538 & 1.0180 & 1.022287230 & 0.004287230 & 6.188722436 & 0.025954036 \\
\hline 6 & 7.2364 & 1.0155 & 1.019923904 & 0.004423904 & 7.380577341 & 0.032013141 \\
\hline 7 & 8.3189 & 1.0140 & 1.016355126 & 0.002355126 & 8.454956661 & 0.019592061 \\
\hline 8 & 9.3097 & 1.0100 & 1.010488234 & 0.000488234 & 9.407342308 & 0.004545308 \\
\hline 9 & 10.2163 & 1.0035 & 1.000622548 & 0.002877452 & 10.22266014 & 0.029396911 \\
\hline 10 & 11.0449 & 0.9880 & 0.984544825 & 0.003455175 & 10.87419914 & 0.038162061 \\
\hline 11 & 11.8018 & 0.9630 & 0.959521942 & 0.003478058 & 11.32408605 & 0.041047349 \\
\hline 12 & 12.4929 & 0.9255 & 0.922843077 & 0.002656923 & 11.52898628 & 0.033192675 \\
\hline 13 & 13.1231 & 0.8725 & 0.872607161 & 0.000107161 & 11.45131103 & 0.001406283 \\
\hline 14 & 13.6983 & 0.8075 & 0.807283342 & 0.000216658 & 11.05840940 & 0.002967848 \\
\hline 15 & 14.2221 & 0.7265 & 0.728345244 & 0.001845244 & 10.35859889 & 0.026243239 \\
\hline 16 & 14.6995 & 0.6345 & 0.637144626 & 0.002644626 & 9.365707433 & 0.038874683 \\
\hline 17 & 15.1346 & 0.5345 & 0.536216243 & 0.001716243 & 8.115418353 & 0.025974653 \\
\hline 18 & 15.5311 & 0.4275 & 0.429510822 & 0.002010822 & 6.670775525 & 0.031230275 \\
\hline 19 & 15.8929 & 0.3185 & 0.318770524 & 0.000270524 & 5.066188057 & 0.004299407 \\
\hline 20 & 16.2229 & 0.2085 & 0.207383220 & 0.001116780 & 3.364357235 & 0.018117415 \\
\hline 21 & 16.5241 & 0.1010 & 0.096159717 & 0.004840283 & 1.588952787 & 0.079981313 \\
\hline 22 & 16.7987 & -0.0080 & -0.00833096 & 0.000330958 & -0.13994927 & 0.005559666 \\
\hline 23 & 17.0499 & -0.1110 & -0.11093900 & $6.09964 E-05$ & -1.89149892 & 0.001039982 \\
\hline 24 & 17.2793 & -0.2090 & -0.20924515 & 0.000245153 & -3.61560977 & 0.004236069 \\
\hline 25 & 17.4885 & -0.3030 & -0.30085484 & 0.002145160 & -5.26149987 & 0.037515629 \\
\hline Sum of IAE & NA & NA & NA & 0.048918 & NA & 0.517259 \\
\hline
\end{tabular}

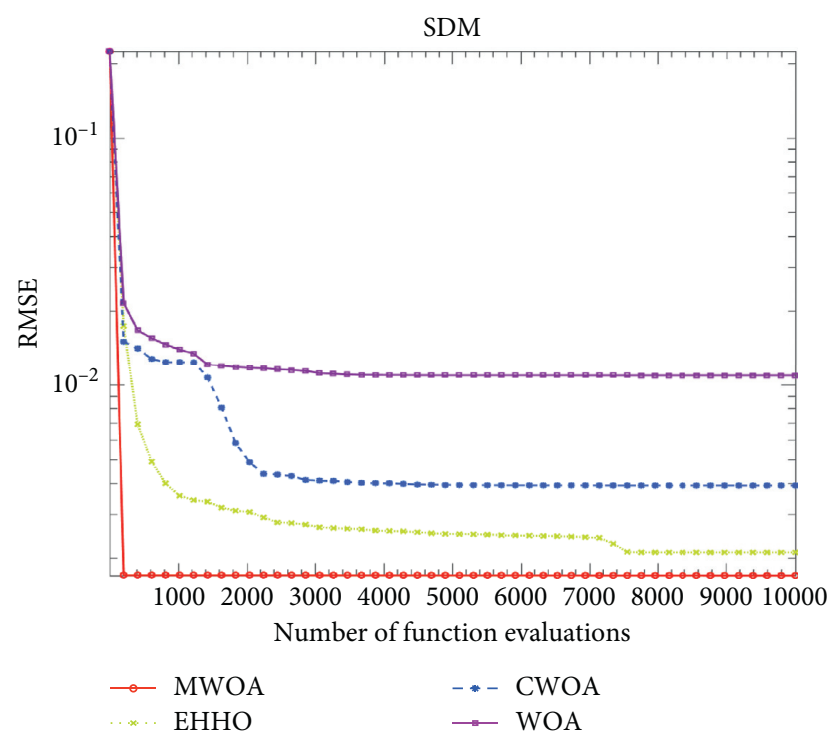

(a)

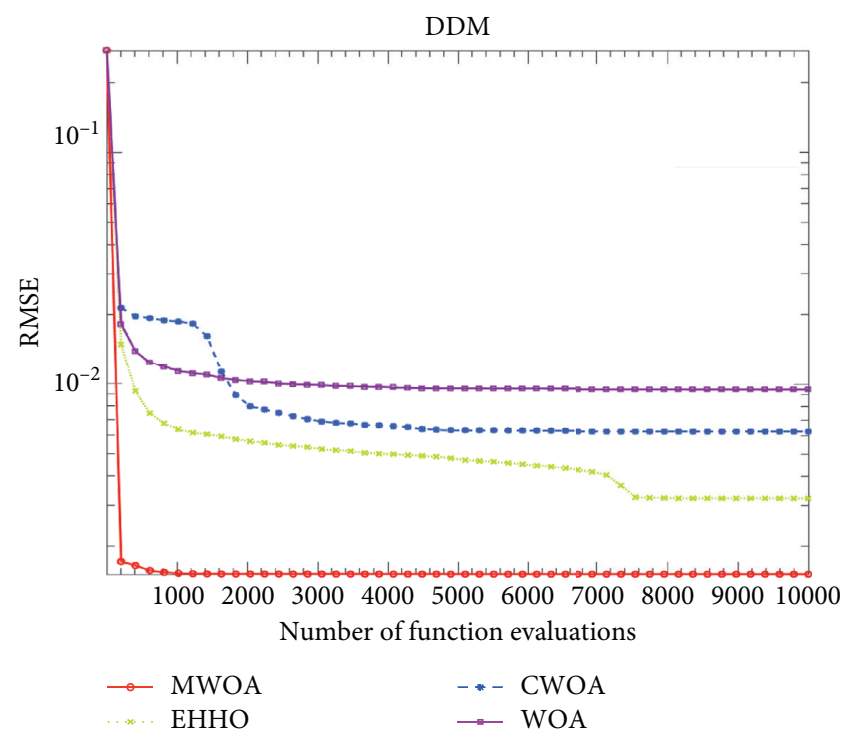

(b)

FIgURE 11: Continued. 


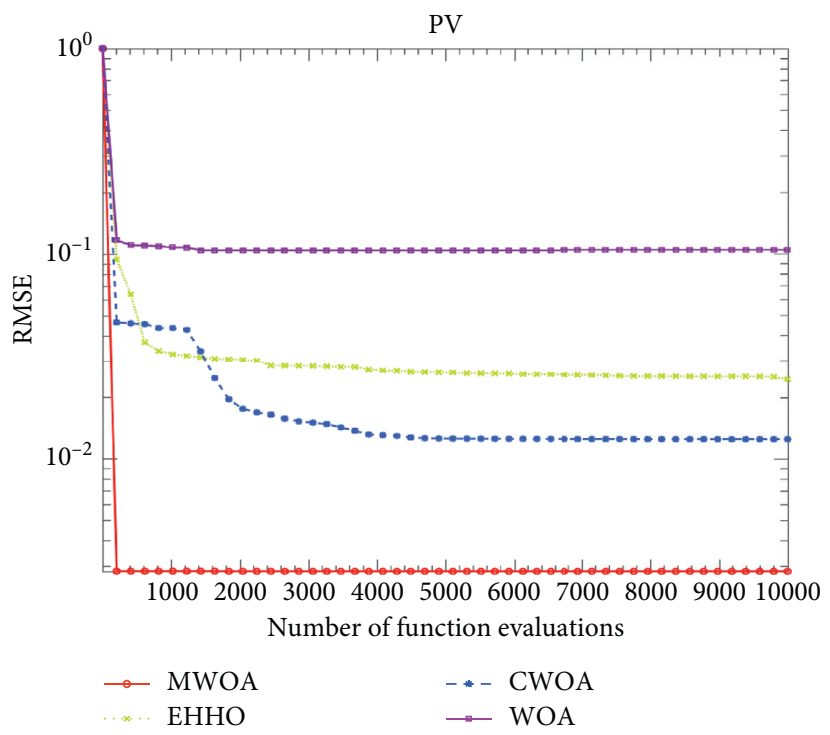

(c)

FIGURE 11: Convergence graphs of MWOA for three models: (a) SDM; (b) DDM; (c) PV.

TABLE 8: Comparisons of MWOA with its variants for three models.

\begin{tabular}{lcccccc}
\hline Model & Algorithm & $\max$ & $\min$ & mean & std & timeCost (ms) \\
\hline \multirow{3}{*}{ SDM } & MWOA & 0.00560131 & $\mathbf{0 . 0 0 0 9 8 6 0 9}$ & $\mathbf{0 . 0 0 1 8 0 8 8 0}$ & 0.00091696 & 1308 \\
& LWOA & 0.04599369 & 0.00112371 & 0.01627346 & 0.01822288 & $\mathbf{1 9 1}$ \\
& PSWOA & $\mathbf{0 . 0 0 3 7 5 8 4 2}$ & 0.00098643 & 0.00176644 & $\mathbf{0 . 0 0 0 5 7 6 2 8}$ & 1284 \\
\hline \multirow{3}{*}{ DDM } & MWOA & $\mathbf{0 . 0 0 2 6 3 3 9}$ & $\mathbf{0 . 0 0 0 9 8 6 0 2}$ & $\mathbf{0 . 0 0 1 3 7 7 1 4}$ & $\mathbf{0 . 0 0 0 5 0 2 4 1}$ & 4318 \\
& LWOA & 0.04601357 & 0.00107692 & 0.00953996 & 0.01368628 & $\mathbf{2 0 5}$ \\
& PSWOA & 0.00369537 & 0.00098964 & 0.00177870 & 0.00075721 & 3298 \\
\multirow{3}{*}{ PV } & MWOA & $\mathbf{0 . 0 0 9 4 3 0 4}$ & 0.00242787 & $\mathbf{0 . 0 0 3 4 3 3 2 2}$ & $\mathbf{0 . 0 0 1 6 7 5 2 3}$ & 0.12821913 \\
& LWOA & 0.27431195 & 0.00251422 & 0.11064789 & 0.00203750 & 1816 \\
\hline
\end{tabular}

5.4. Results of ST40 and SM55. To further test the utility of the method proposed in this paper, we apply MWOA to parameter extraction in the two different types of $\mathrm{PV}$ modules: thin-film PV module ST40 [84] and monocrystalline silicon PV module SM55 [85]. Both the two PV modules are modeled by SDM and DDM. For each module type, experiments were conducted at different temperatures and different light intensities. When the temperature changes, the light intensity needs to keep constant; conversely, when the light intensity changes, the temperature keeps constant.

The initial range of the photogenic current $I_{\mathrm{ph}}$ in this part is determined by the short circuit current $I_{\mathrm{SC}}$ and its temperature coefficient $\alpha$ under standard test conditions (STC). The calculation of $I_{\mathrm{ph}}$ is as follows:

$$
I_{\mathrm{SC}}(G, T)=I_{\mathrm{SC} \_ \text {STC }} \cdot \frac{G}{G_{\mathrm{STC}}}+\alpha \cdot\left(T-T_{\mathrm{STC}}\right),
$$

TABLE 9: Ranges of parameters for ST40 and SM55.

\begin{tabular}{lccccc}
\hline Parameters & $I_{\mathrm{ph}}(A)$ & $I_{\mathrm{sd}}(A)$ & $R_{s}(\Omega)$ & $R_{p}(\Omega)$ & $n$ \\
\hline Lower limit & 0 & 0 & 0 & 0 & 1 \\
Upper limit & $2 I_{\mathrm{SC}}$ & $100 e-6$ & 2 & 5000 & 4 \\
\hline
\end{tabular}

where $G$ and $T$ represent the light intensity and the temperature under non-STC, respectively. Thus, the parameter ranges are given in Table 9.

Tables 10 and 11 give the two PV modules' best parameter values in SDM and DDM extracted by MWOA at different irradiances. In addition, their values of parameters under different temperature conditions are listed in Tables 12 and 13. Furthermore, Figures 12 and 13 present the results of the comparison between the simulated current and experimental data at various irradiances for both SDM and DDM, while comparative results at different temperatures 
TABle 10: Parameter estimates by MWOA for ST40 at different irradiances with a temperature of $25^{\circ} \mathrm{C}$.

\begin{tabular}{|c|c|c|c|c|c|}
\hline \multirow{2}{*}{ Parameter } & \multicolumn{5}{|c|}{ Irradiance $\left(\mathrm{W} / \mathrm{m}^{2}\right)$} \\
\hline & 200 & 400 & 600 & 800 & 1000 \\
\hline \multicolumn{6}{|l|}{$S D M$} \\
\hline$I_{\mathrm{Ph}}(\mathrm{A})$ & 0.53331757 & 1.066988270 & 1.60810360 & 2.134525548 & 2.672172239 \\
\hline$I_{\mathrm{sd}}(\mathrm{mA})$ & $1.26871 E-06$ & $2.25447 E-06$ & $5.7171 E-07$ & $2.13585 E-06$ & $2.49412 E-06$ \\
\hline$R_{s}(\Omega)$ & 1.246907638 & 1.044231634 & 1.22988867 & 1.072832043 & 1.077531612 \\
\hline$R_{\mathrm{sh}}(\Omega)$ & 342.7239747 & 373.8873942 & 301.602681 & 396.1091591 & 431.4419003 \\
\hline$n$ & 1.731262353 & 1.805362555 & 1.63808006 & 1.794001397 & 1.811129005 \\
\hline RMSE & 0.000482114 & 0.000719576 & 0.00197090 & 0.002108032 & 0.001978270 \\
\hline \multicolumn{6}{|l|}{$D D M$} \\
\hline$I_{\mathrm{Ph}}(\mathrm{A})$ & 0.533334706 & 1.067494405 & 1.603128611 & 2.135349650 & 2.672683458 \\
\hline$I_{\mathrm{sd}_{1}}(\mathrm{~mA})$ & $3.18603 E-05$ & $1.10843 E-05$ & $2.01305 E-07$ & $2.63126 E-05$ & $3.68549 E-07$ \\
\hline$I_{\mathrm{sd}_{2}}(\mathrm{~mA})$ & $1.82510 E-07$ & $1.73461 E-06$ & $9.10872 E-05$ & $9.23098 E-08$ & $6.39422 E-05$ \\
\hline$R_{s}(\Omega)$ & 1.607320066 & 1.086406066 & 1.228284977 & 1.193810586 & 1.154094959 \\
\hline$R_{\mathrm{sh}}(\Omega)$ & 362.7821760 & 365.4957689 & 424.7460606 & 411.8634978 & 463.7373381 \\
\hline$n_{1}$ & 2.954593428 & 3.999998684 & 1.538718540 & 2.581648985 & 1.601798267 \\
\hline$n_{2}$ & 1.517748630 & 1.770462365 & 3.259517500 & 1.479398414 & 3.062640621 \\
\hline RMSE & 0.000464494 & 0.000640640 & 0.001199160 & 0.001561701 & 0.001413850 \\
\hline
\end{tabular}

TABle 11: Parameter estimates by MWOA for SM55 at different irradiances with a temperature of $25^{\circ} \mathrm{C}$.

\begin{tabular}{|c|c|c|c|c|c|}
\hline \multirow{2}{*}{ Parameter } & \multicolumn{5}{|c|}{ Irradiance $\left(\mathrm{W} / \mathrm{m}^{2}\right)$} \\
\hline & 200 & 400 & 600 & 800 & 1000 \\
\hline \multicolumn{6}{|l|}{$S D M$} \\
\hline$I_{\mathrm{Ph}}(\mathrm{A})$ & 0.691124151 & 1.375557785 & 2.060699041 & 2.754700747 & 3.445108764 \\
\hline$I_{\mathrm{sd}}(\mathrm{mA})$ & $3.14217 E-07$ & $2.59878 E-06$ & $5.59042 E-06$ & $8.35283 E-07$ & $5.71094 E-06$ \\
\hline$R_{s}(\Omega)$ & 0.045346426 & 0 & 0.025746037 & 0.226456305 & 0.168590083 \\
\hline$R_{\mathrm{sh}}(\Omega)$ & 460.8732310 & 982.5299237 & 3737.137718 & 826.8341087 & 5000 \\
\hline$n$ & 1.450790398 & 1.679741930 & 1.769714298 & 1.539436492 & 1.761010518 \\
\hline RMSE & $7.33928 E-04$ & $5.30961 E-03$ & $9.59915 E-03$ & $5.03150 E-03$ & $1.95455 E-02$ \\
\hline \multicolumn{6}{|l|}{$D D M$} \\
\hline$I_{\mathrm{Ph}}(\mathrm{A})$ & 0.691774691 & 1.382238669 & 2.069823060 & 2.760191237 & 3.444815843 \\
\hline$I_{\mathrm{sd}_{1}}(\mathrm{~mA})$ & $2.67313 E-05$ & $6.75894 E-05$ & $3.32656 E-05$ & $1.18388 E-07$ & $4.47349 E-07$ \\
\hline$I_{\mathrm{sd}_{2}}(\mathrm{~mA})$ & $1.21842 E-07$ & $7.43739 E-08$ & $1.83266 E-07$ & $2.02677 E-05$ & $3.20927 E-08$ \\
\hline$R_{s}(\Omega)$ & 0.301600858 & 0.410528644 & 0.315554320 & 0.344567527 & 0.294658909 \\
\hline$R_{\mathrm{sh}}(\Omega)$ & 452.4222904 & 465.8227848 & 500.5793324 & 475.8515534 & 835.4957903 \\
\hline$n_{1}$ & 3.999998927 & 3.999999857 & 3.999999242 & 1.365722181 & 1.479773104 \\
\hline$n_{2}$ & 1.364883480 & 1.328568747 & 1.401671201 & 3.530470772 & 3.999998105 \\
\hline RMSE & $5.55361 E-04$ & $6.42907 E-03$ & $1.13452 E-03$ & $7.36226 E-04$ & $4.85349 E-03$ \\
\hline
\end{tabular}

TABLE 12: The optimal extracted parameters for SM55 based on MWOA at various temperatures with irradiance of $1000\left(\mathrm{~W} / \mathrm{m}^{2}\right)$.

\begin{tabular}{|c|c|c|c|c|}
\hline \multirow{2}{*}{ Parameter } & \multicolumn{4}{|c|}{ Temperature } \\
\hline & $25^{\circ} \mathrm{C}$ & $40^{\circ} \mathrm{C}$ & $50^{\circ} \mathrm{C}$ & $70^{\circ} \mathrm{C}$ \\
\hline \multicolumn{5}{|l|}{$S D M$} \\
\hline$I_{\mathrm{Ph}}(\mathrm{A})$ & 2.670805171 & 2.678136578 & 2.692226584 & 2.692391664 \\
\hline$I_{\mathrm{sd}}(\mathrm{mA})$ & $2.99498 E-06$ & $7.45505 E-06$ & $1.82816 E-05$ & $8.72477 E-05$ \\
\hline$R_{s}(\Omega)$ & 1.063555711 & 1.109929841 & 1.151349018 & 1.126145392 \\
\hline$R_{\mathrm{sh}}(\Omega)$ & 468.8521810 & 425.9939767 & 291.6453129 & 366.3672871 \\
\hline$n$ & 1.834954118 & 1.759078925 & 1.714508611 & 1.726804626 \\
\hline RMSE & $2.63628 E-03$ & $1.823996 E-03$ & $1.825667 E-03$ & $7.77905 E-04$ \\
\hline \multicolumn{5}{|l|}{$D D M$} \\
\hline$I_{\mathrm{Ph}}(\mathrm{A})$ & 2.671398029 & 2.679666230 & 2.689671608 & 2.692165113 \\
\hline$I_{\mathrm{sd} 1}(\mathrm{~mA})$ & $2.11052 E-06$ & $4.11310 E-06$ & $6.53862 E-06$ & $4.07806 E-05$ \\
\hline$I_{\mathrm{sd} 2}(\mathrm{~mA})$ & $5.16008 E-05$ & $9.13747 E-05$ & $7.44782 E-05$ & $5.01099 E-05$ \\
\hline$R_{s}(\Omega)$ & 1.083233193 & 1.139556934 & 1.170833251 & 1.126314671 \\
\hline$R_{\mathrm{sh}}(\Omega)$ & 465.8813651 & 409.7291090 & 350.2016170 & 372.8377988 \\
\hline$n_{1}$ & 1.790806924 & 1.683990294 & 1.596889739 & 1.687289644 \\
\hline$n_{2}$ & 4 & 3.459103314 & 2.383804127 & 1.787270097 \\
\hline RMSE & $2.026022 E-03$ & $1.434158 E-03$ & $1.691410 E-03$ & $7.86324 E-03$ \\
\hline
\end{tabular}


TABLE 13: The optimal extracted parameters for SM55 based on MWOA at various temperatures with irradiance of $1000\left(\mathrm{~W} / \mathrm{m}^{2}\right)$.

\begin{tabular}{lccc}
\hline Parameter & & Temperature & \\
& $25^{\circ} \mathrm{C}$ & $40^{\circ} \mathrm{C}$ & $60^{\circ} \mathrm{C}$ \\
\hline$S D M$ & & & 3.496202107 \\
$I_{\mathrm{Ph}}(\mathrm{A})$ & 3.439753496 & 3.464789448 & $5.76199 E-06$ \\
$I_{\mathrm{sd}}(\mathrm{mA})$ & $1.09955 E-06$ & $2.12791 E-06$ & 0.326680838 \\
$R_{s}(\Omega)$ & 0.258317606 & 0.288320732 & 420.4346275 \\
$R_{\mathrm{sh}}(\Omega)$ & 3185.898949 & 920.0665445 & 1.386130001 \\
$n$ & 1.568081730 & 1.478821787 & $3.943069 E-03$ \\
$\mathrm{RMSE}$ & $9.36221 E-03$ & $5.238358 E-03$ & 3.494227304 \\
$D D M$ & & & $5.56214 E-06$ \\
$I_{\mathrm{Ph}}(\mathrm{A})$ & 3.444339148 & 3.468216376 & $9.81529 E-05$ \\
$I_{\text {sd } 1}(\mathrm{~mA})$ & $4.84749 E-07$ & $1.69984 E-06$ & 0.324250130 \\
$I_{\text {sd2 }}(\mathrm{mA})$ & $1.48188 E-06$ & $1.30229 E-06$ & 535.3914659 \\
$R_{s}(\Omega)$ & 0.291523040 & 0.308086727 & 1.383430623 \\
$R_{\mathrm{sh}}(\Omega)$ & 897.8080508 & 584.7482411 & 3.060301029 \\
$n_{1}$ & 1.487262815 & 3.999999340 & $3.890039 E-03$ \\
$n_{2}$ & 4 & 1.430089460 & $3.865419 E-03$ \\
$\mathrm{RMSE}$ & $5.262165 E-03$ & & \\
\hline
\end{tabular}

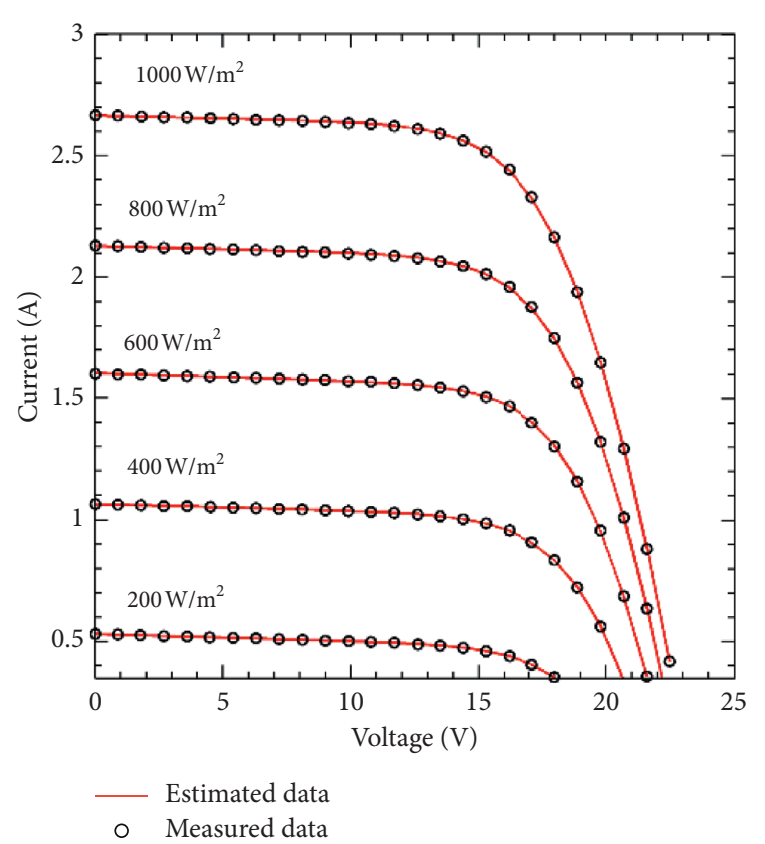

(a)

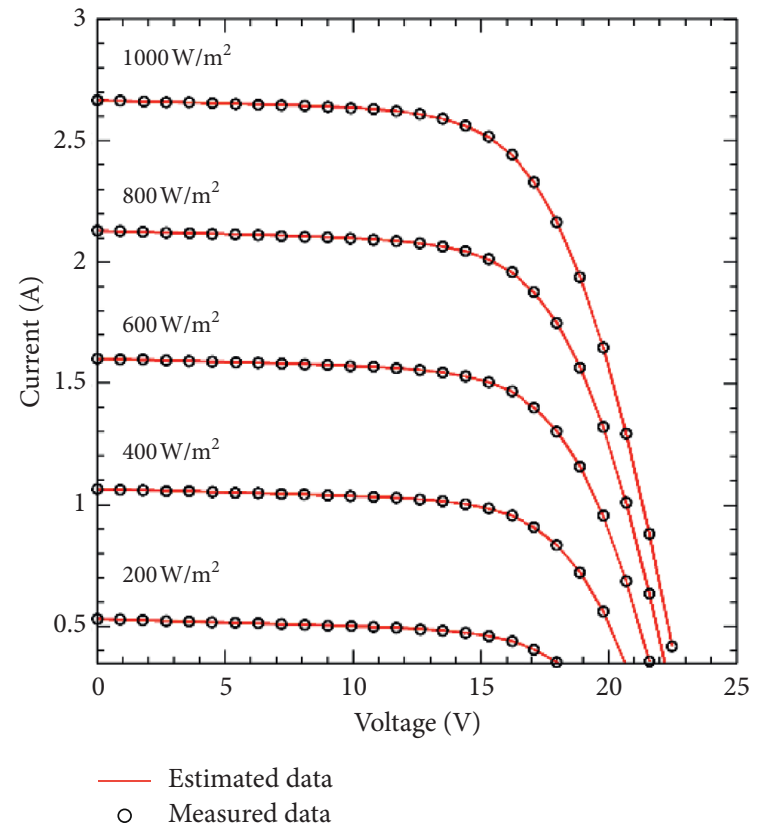

(b)

FIgURE 12: I-VC of (a) SDM and (b) DDM for thin-film ST40 at different irradiances. 


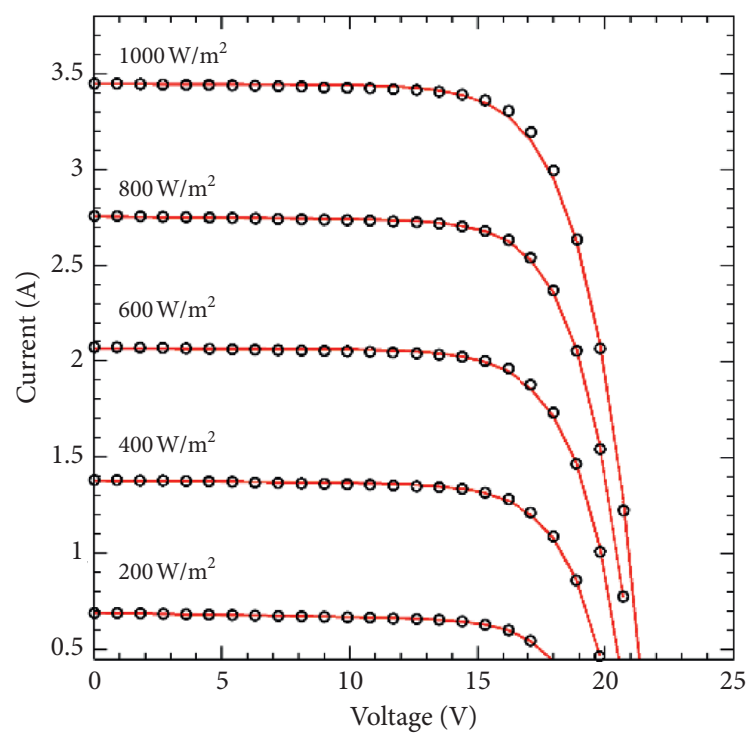

Estimated data

- Measured data

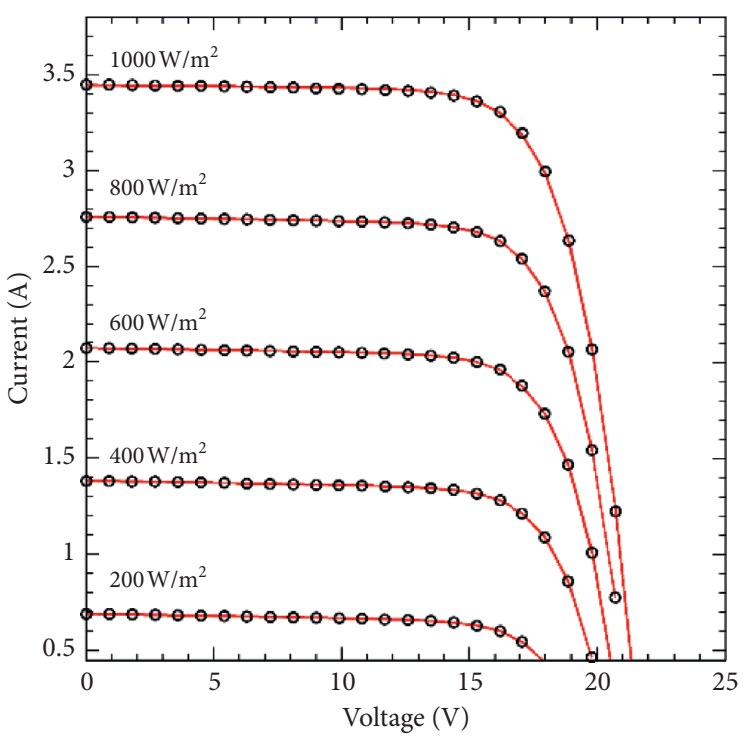

Estimated data

- Measured data

(a)

(b)

FIGURE 13: I-VC of (a) SDM and (b) DDM for monocrystalline SM55 at different irradiances.

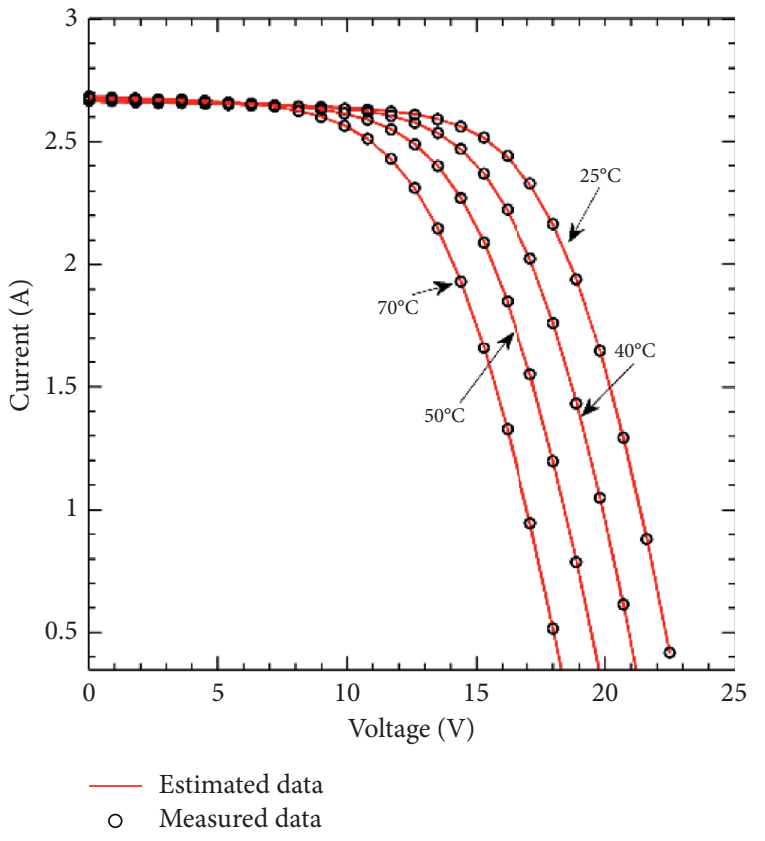

(a)

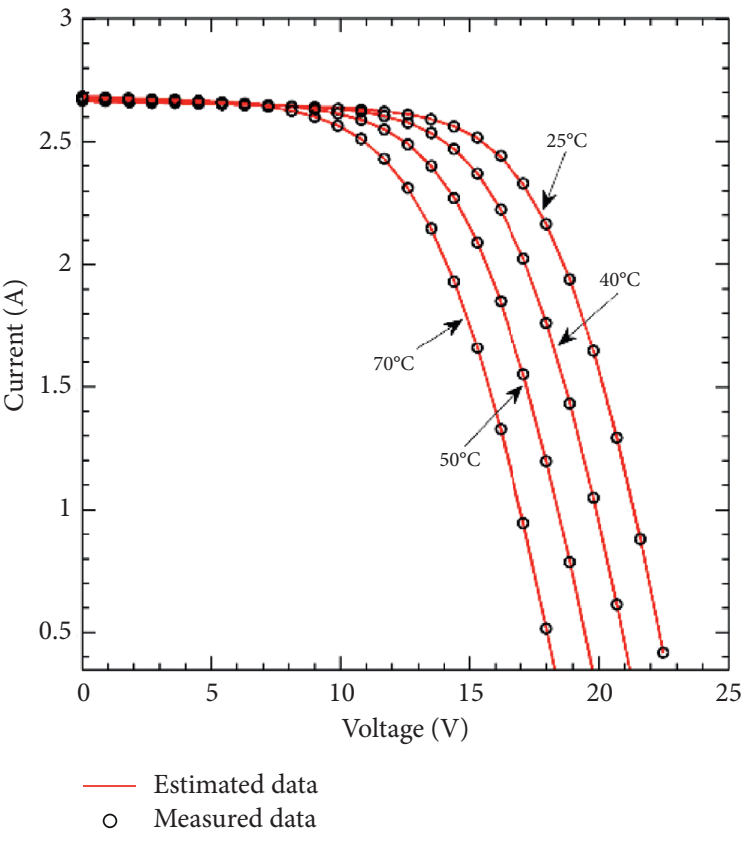

(b)

FIgURE 14: I-VC of (a) SDM and (b) DDM for thin-film ST40 at different temperatures. 


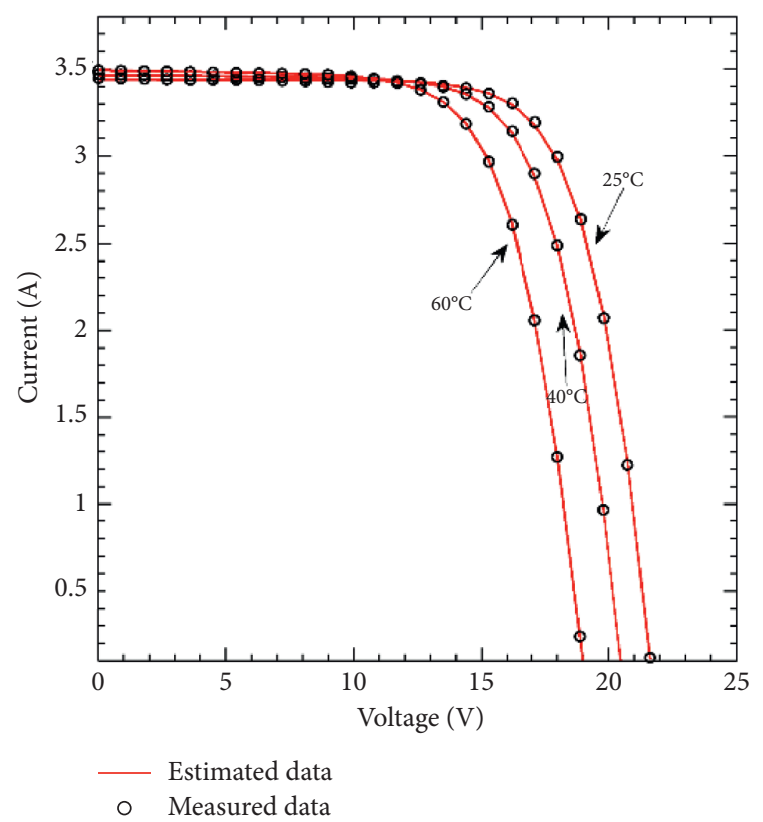

(a)

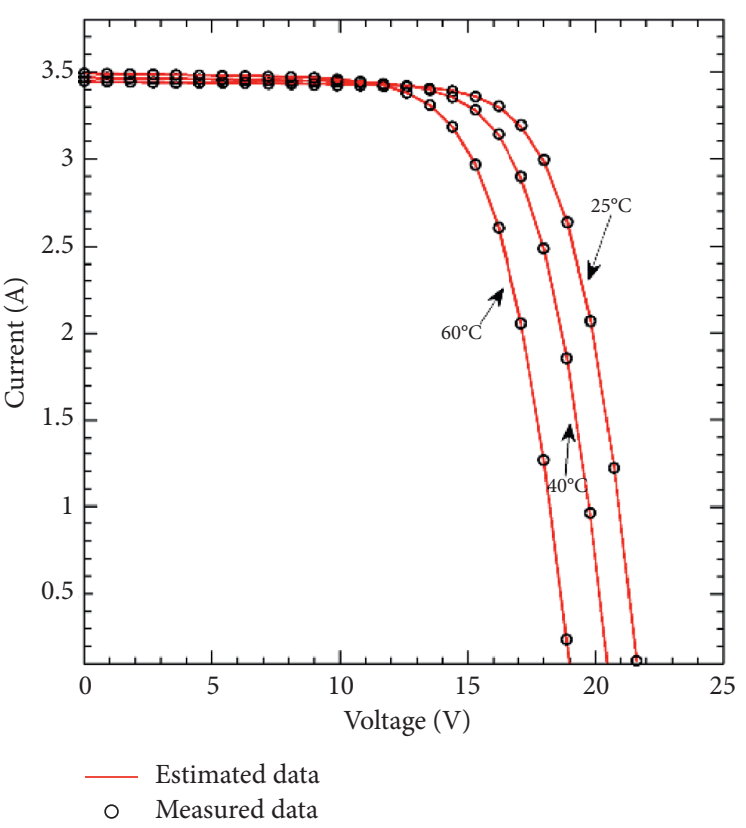

(b)

FIGURE 15: I-VC of (a) SDM and (b) DDM for monocrystalline SM55 at different temperatures.

are given in Figures 14 and 15. It can be seen from the figures that the two PV modules' I-VCs of the two models estimated by MWOA are both in good accordance with the experimental data no matter under different irradiance or temperature conditions.

\section{Conclusions and Future Directions}

In this paper, we propose a modified whale optimization algorithm for identifying the unknown parameters in the optimal models which characterize solar cells and photovoltaic modules. The new algorithm MWOA is formed by introducing Levy flight strategy and pattern search mechanism. MWOA follows the population update approach of the original WOA to find the current optimal solution which is used as the initial point of pattern search; then, it searches for potential better solutions in the domain of this solution. Meanwhile, it makes use of Levy flight to maintain the diversity of solutions; thus, it enhances the ability to explore the entire search space. These two improvement mechanisms balance the relationship between exploration and exploitation and allow the algorithm to increase the solution accuracy, while they mitigate the possibility of the algorithm falling into local optimization. Moreover, we demonstrate the validity and the utility of the proposed method in the problem of identifying parameters of solar cell and PV modules by analyzing the experimental results.

There are still interesting aspects that can be further explored in future work. Firstly, we have utilized MWOA to obtain satisfactory results in parameter estimation for solar cells and PV modules, and we will investigate its application to other problems including engineering application [86], multithreshold image segmentation $[87,88]$, bankruptcy prediction [89], engineering design [65, 90-93], financial stress prediction [94], constrained practical engineering problems [95], support vector machines [66, 96, 97], extreme learning machine (ELM) [98-103], convolutional neural networks [104-107], medical image recognition [108], feature selection [66, 109-113], feature information fusion [114], social evolution modeling [115], recommender system [116], text clustering [117], and unsupervised band selection [118]. Secondly, since MWOA is a population-based stochastic optimizer that may still encounter population stagnation problems for some more complex datasets, further improvement of the algorithm performance based on other optimization mechanisms is also a meaningful research direction.

\section{Data Availability}

The data involved in this study are all public data, which can be downloaded through public channels.

\section{Conflicts of Interest}

The authors declare that there are no conflicts of interest regarding the publication of the article.

\section{Authors' Contributions}

Chen Chi, Huiling Chen, and Hailong Huang contributed equally to this work.

\section{Acknowledgments}

This study was supported by the National Social Science Fund of China, "Research on the Coordination between Distributive Justice and Economic Efficiency in the Process 
of New Urbanization" (Project no. 16CTJ012). Thanks are due to Ali Asghar Heidari for his efforts during the preparation of this research.

\section{References}

[1] I. Nassar-eddine, A. Obbadi, Y. Errami, A. El fajri, and M. Agunaou, "Parameter estimation of photovoltaic modules using iterative method and the Lambert W function: a comparative study," Energy Conversion and Management, vol. 119, pp. 37-48, 2016.

[2] B. Parida, S. Iniyan, and R. Goic, "A review of solar photovoltaic technologies," Renewable and Sustainable Energy Reviews, vol. 15, no. 3, pp. 1625-1636, 2011.

[3] A. Askarzadeh and A. Rezazadeh, "Artificial bee swarm optimization algorithm for parameters identification of solar cell models," Applied Energy, vol. 102, pp. 943-949, 2013.

[4] D. F. Alam, D. A. Yousri, and M. B. Eteiba, "Flower Pollination Algorithm based solar PV parameter estimation," Energy Conversion and Management, vol. 101, pp. 410-422, 2015.

[5] D. S. H. Chan, J. R. Phillips, and J. C. H. Phang, "A comparative study of extraction methods for solar cell model parameters," Solid-State Electronics, vol. 29, no. 3, pp. 329-337, 1986.

[6] Z. Ouennoughi and M. Chegaar, "A simpler method for extracting solar cell parameters using the conductance method," Solid-State Electronics, vol. 43, no. 11, pp. 1985-1988, 1999.

[7] M. Chegaar, Z. Ouennoughi, and A. Hoffmann, "A new method for evaluating illuminated solar cell parameters," Solid-State Electronics, vol. 45, no. 2, pp. 293-296, 2001.

[8] A. Jain and A. Kapoor, "Exact analytical solutions of the parameters of real solar cells using Lambert W-function," Solar Energy Materials and Solar Cells, vol. 81, no. 2, pp. 269-277, 2004.

[9] F. Khan, S. N. Singh, and M. Husain, "Effect of illumination intensity on cell parameters of a silicon solar cell," Solar Energy Materials and Solar Cells, vol. 94, no. 9, pp. 14731476, 2010.

[10] N. Santakrus Singh, A. Jain, and A. Kapoor, "Determination of the solar cell junction ideality factor using special trans function theory (STFT)," Solar Energy Materials and Solar Cells, vol. 93, no. 8, pp. 1423-1426, 2009.

[11] S. P. Mallick, D. P. Dash, S. Mallik et al., "An empirical approach towards photovoltaic parameter extraction and optimization," Solar Energy, vol. 153, pp. 360-365, 2017.

[12] Q. Wu, T. Song, H. Liu, and X. Yan, "Particle swarm optimization algorithm based on parameter improvements," Journal of Computational Methods in Sciences and Engineering, vol. 17, no. 3, pp. 557-568, 2017.

[13] C. Lin, A. Qing, and Q. Feng, "A comparative study of crossover in differential evolution," Journal of Heuristics, vol. 17 , no. 6 , pp. 675-703, 2011.

[14] R. Salgotra and U. Singh, "The naked mole-rat algorithm," Neural Computing and Applications, vol. 31, no. 12, pp. 8837-8857, 2019.

[15] A. A. Heidari, S. Mirjalili, H. Faris, I. Aljarah, M. Mafarja, and $\mathrm{H}$. Chen, "Harris hawks optimization: algorithm and applications," Future Generation Computer Systems, vol. 97, pp. 849-872, 2019.

[16] S. Li, H. Chen, M. Wang, A. A. Heidari, and S. Mirjalili, "Slime mould algorithm: a new method for stochastic optimization," Future Generation Computer Systems, vol. 111, pp. 300-323, 2020.

[17] H. Chen, S. Jiao, A. A. Heidari, M. Wang, X. Chen, and $\mathrm{X}$. Zhao, "An opposition-based sine cosine approach with local search for parameter estimation of photovoltaic models," Energy Conversion and Management, vol. 195, pp. 927-942, 2019.

[18] H. Chen, S. Jiao, M. Wang, A. A. Heidari, and X. Zhao, "Parameters identification of photovoltaic cells and modules using diversification-enriched Harris hawks optimization with chaotic drifts," Journal of Cleaner Production, vol. 244, Article ID 118778, 2020.

[19] H. M. Ridha, A. A. Heidari, M. Wang, and H. Chen, "Boosted mutation-based Harris hawks optimizer for parameters identification of single-diode solar cell models," Energy Conversion and Management, vol. 209, Article ID 112660, 2020.

[20] H. M. Ridha, C. Gomes, H. Hizam, M. Ahmadipour, A. A. Heidari, and H. Chen, "Multi-objective optimization and multi-criteria decision-making methods for optimal design of standalone photovoltaic system: a comprehensive review," Renewable and Sustainable Energy Reviews, vol. 135, Article ID 110202, 2021.

[21] S. Jiao, G. Chong, C. Huang et al., "Orthogonally adapted Harris hawks optimization for parameter estimation of photovoltaic models," Energy, vol. 203, Article ID 117804, 2020.

[22] H. Zhang, R. Li, Z. Cai et al., "Advanced orthogonal moth flame optimization with Broyden-Fletcher-Goldfarb-Shanno algorithm: framework and real-world problems," Expert Systems with Applications, vol. 159, Article ID 113617, 2020.

[23] Y. Liu, G. Chong, A. A. Heidari et al., "Horizontal and vertical crossover of Harris hawk optimizer with NelderMead simplex for parameter estimation of photovoltaic models," Energy Conversion and Management, vol. 223, Article ID 113211, 2020

[24] M. Wang, X. Zhao, A. A. Heidari, and H. Chen, "Evaluation of constraint in photovoltaic models by exploiting an enhanced ant lion optimizer," Solar Energy, vol. 211, pp. 503-521, 2020.

[25] R. B. Messaoud, "Extraction of uncertain parameters of single and double diode model of a photovoltaic panel using Salp Swarm algorithm," Measurement, vol. 154, Article ID 107446, 2020.

[26] R. Abbassi, A. Abbassi, A. A. Heidari, and S. Mirjalili, "An efficient salp swarm-inspired algorithm for parameters identification of photovoltaic cell models," Energy Conversion and Management, vol. 179, pp. 362-372, 2019.

[27] A. Abbassi, R. Abbassi, A. A. Heidari et al., "Parameters identification of photovoltaic cell models using enhanced exploratory salp chains-based approach," Energy, vol. 198, Article ID 117333, 2020.

[28] N. Pourmousa, S. M. Ebrahimi, M. Malekzadeh, and M. Alizadeh, "Parameter estimation of photovoltaic cells using improved Lozi map based chaotic optimization Algorithm," Solar Energy, vol. 180, pp. 180-191, 2019.

[29] X. Chen, B. Xu, C. Mei, Y. Ding, and K. Li, "Teachinglearning-based artificial bee colony for solar photovoltaic parameter estimation," Applied Energy, vol. 212, pp. 15781588, 2018.

[30] S. Li, W. Gong, X. Yan et al., "Parameter extraction of photovoltaic models using an improved teaching-learningbased optimization," Energy Conversion and Management, vol. 186, pp. 293-305, 2019. 
[31] J. Liang, K. Qiao, M. Yuan et al., "Evolutionary multi-task optimization for parameters extraction of photovoltaic models," Energy Conversion and Management, vol. 207, Article ID 112509, 2020.

[32] S. Li, Q. Gu, W. Gong, and B. Ning, “An enhanced adaptive differential evolution algorithm for parameter extraction of photovoltaic models," Energy Conversion and Management, vol. 205, Article ID 112443, 2020.

[33] S. Li, W. Gong, X. Yan, C. Hu, D. Bai, and L. Wang, "Parameter estimation of photovoltaic models with memetic adaptive differential evolution," Solar Energy, vol. 190, pp. 465-474, 2019.

[34] X. Chen and K. Yu, "Hybridizing cuckoo search algorithm with biogeography-based optimization for estimating photovoltaic model parameters," Solar Energy, vol. 180, pp. 192-206, 2019.

[35] D. Yousri, D. Allam, M. B. Eteiba, and P. N. Suganthan, "Static and dynamic photovoltaic models' parameters identification using chaotic heterogeneous comprehensive learning particle swarm optimizer variants," Energy Conversion and Management, vol. 182, pp. 546-563, 2019.

[36] J. Liang, S. Ge, B. Qu et al., "Classified perturbation mutation based particle swarm optimization algorithm for parameters extraction of photovoltaic models," Energy Conversion and Management, vol. 203, Article ID 112138, 2020.

[37] X. Lin and Y. Wu, "Parameters identification of photovoltaic models using niche-based particle swarm optimization in parallel computing architecture," Energy, vol. 196, Article ID 117054, 2020.

[38] X. Jian and Z. Weng, "A logistic chaotic JAYA algorithm for parameters identification of photovoltaic cell and module models," Optik, vol. 203, Article ID 164041, 2020.

[39] Y. Zhang, Z. Jin, X. Zhao, and Q. Yang, "Backtracking search algorithm with Lévy flight for estimating parameters of photovoltaic models," Energy Conversion and Management, vol. 208, Article ID 112615, 2020.

[40] H. Zhang, A. A. Heidari, M. Wang, L. Zhang, H. Chen, and C. Li, "Orthogonal Nelder-Mead moth flame method for parameters identification of photovoltaic modules," Energy Conversion and Management, vol. 211, Article ID 112764, 2020.

[41] K. b. o. Medani, S. Sayah, and A. Bekrar, "Whale optimization algorithm based optimal reactive power dispatch: a case study of the Algerian power system," Electric Power Systems Research, vol. 163, pp. 696-705, 2018.

[42] I. Aljarah, H. Faris, and S. Mirjalili, "Optimizing connection weights in neural networks using the whale optimization algorithm," Soft Computing, vol. 22, no. 1, pp. 1-15, 2018.

[43] M. A. E. Aziz, A. A. Ewees, and A. E. Hassanien, "Whale optimization algorithm and moth-flame optimization for multilevel thresholding image segmentation," Expert Systems with Applications, vol. 83, pp. 242-256, 2017.

[44] M. M. Mafarja and S. Mirjalili, "Hybrid Whale Optimization Algorithm with simulated annealing for feature selection," Neurocomputing, vol. 260, pp. 302-312, 2017.

[45] J. Wang, P. Du, T. Niu, and W. Yang, "A novel hybrid system based on a new proposed algorithm-Multi-Objective Whale Optimization Algorithm for wind speed forecasting," Applied Energy, vol. 208, pp. 344-360, 2017.

[46] R. Salgotra, U. Singh, and S. Saha, "On some improved versions of whale optimization algorithm," Arabian Journal for Science And Engineering, vol. 44, no. 11, pp. 9653-9691, 2019.
[47] H. Chen, C. Yang, A. A. Heidari, and X. Zhao, "An efficient double adaptive random spare reinforced whale optimization algorithm," Expert Systems with Applications, vol. 154, Article ID 113018, 2020.

[48] H. Ding, Z. Wu, and L. Zhao, "Whale optimization algorithm based on nonlinear convergence factor and chaotic inertial weight," Concurrency and Computation-Practice \& Experience, vol. 32, no. 24, Article ID e5949, 2020.

[49] Y. Ling, Y. Zhou, and Q. Luo, "Lévy flight trajectory-based whale optimization algorithm for global optimization," IEEE Access, vol. 5, pp. 6168-6186, 2017.

[50] Y. Zhou, Y. Niu, Q. Luo, and M. Jiang, “Teaching learningbased whale optimization algorithm for multi-layer perceptron neural network training," Mathematical Biosciences and Engineering, vol. 17, no. 5, pp. 5987-6025, 2020.

[51] Y. Niu, Y. Zhou, and Q. Luo, "Optimize star sensor calibration based on integrated modeling with hybrid WOA-LM algorithm," Journal of Intelligent \& Fuzzy Systems, vol. 38, no. 3, pp. 2683-2691, 2020.

[52] Q. Luo, Y. Ling, and Y. Zhou, "Modified whale optimization algorithm for infinitive impulse response system identification," Arabian Journal for Science and Engineering, vol. 45, no. 3, pp. 2163-2176, 2020.

[53] W. Long, T. Wu, J. Jiao, M. Tang, and M. Xu, "Refractionlearning-based whale optimization algorithm for high-dimensional problems and parameter estimation of PV model," Engineering Applications of Artificial Intelligence, vol. 89, Article ID 103457, 2020.

[54] G. Xiong, J. Zhang, D. Shi, and Y. He, "Parameter extraction of solar photovoltaic models using an improved whale optimization algorithm," Energy Conversion and Management, vol. 174, pp. $388-405,2018$

[55] O. S. Elazab, H. M. Hasanien, M. A. Elgendy, and A. M. Abdeen, "Parameters estimation of single- and multiple-diode photovoltaic model using whale optimisation algorithm," IET Renewable Power Generation, vol. 12, no. 15, pp. 1755-1761, 2018.

[56] D. Oliva, M. Abd El Aziz, and A. Ella Hassanien, "Parameter estimation of photovoltaic cells using an improved chaotic whale optimization algorithm," Applied Energy, vol. 200, pp. 141-154, 2017.

[57] J. Hu, H. Chen, A. A. Heidari et al., "Orthogonal learning covariance matrix for defects of Grey Wolf optimizer: insights, balance, diversity, and feature selection," KnowledgeBased Systems, vol. 213, Article ID 106684, 2020.

[58] Q. Niu, H. Zhang, and K. Li, “An improved TLBO with elite strategy for parameters identification of PEM fuel cell and solar cell models," International Journal of Hydrogen Energy, vol. 39, no. 8, pp. 3837-3854, 2014.

[59] G. Ren, R. Yang, R. Yang, and P. Zheng, "A parameter estimation method for fractional-order nonlinear systems based on improved whale optimization algorithm," Modern Physics Letters B, vol. 33, no. 7, 2019.

[60] W. Guo, T. Liu, F. Dai, and P. Xu, "An improved whale optimization algorithm for feature selection," Computers, Materials \& Continua, vol. 62, no. 1, pp. 337-354, 2020.

[61] R. K. Agrawal, B. Kaur, and S. Sharma, "Quantum based Whale Optimization Algorithm for wrapper feature selection," Applied Soft Computing, vol. 89, 2020.

[62] I. Strumberger, N. Bacanin, M. Tuba, and E. Tuba, "Resource scheduling in cloud computing based on a hybridized whale optimization algorithm," Applied Sciences, vol. 9, no. 22, 2019. 
[63] J. Nasiri and F. M. Khiyabani, "A whale optimization algorithm (WOA) approach for clustering," Cogent Mathematics, vol. 5, no. 1, 2018.

[64] H. Chen, Y. Xu, M. Wang, and X. Zhao, "A balanced whale optimization algorithm for constrained engineering design problems," Applied Mathematical Modelling, vol. 71, pp. 45-59, 2019.

[65] J. Luo, H. Chen, A. A. Heidari, Y. Xu, Q. Zhang, and C. Li, "Multi-strategy boosted mutative whale-inspired optimization approaches," Applied Mathematical Modelling, vol. 73, pp. 109-123, 2019.

[66] M. Wang and H. Chen, "Chaotic multi-swarm whale optimizer boosted support vector machine for medical diagnosis," Applied Soft Computing, vol. 88, Article ID 105946, 2020.

[67] A. A. Heidari, I. Aljarah, H. Faris, H. Chen, J. Luo, and S. Mirjalili, "An enhanced associative learning-based exploratory whale optimizer for global optimization," Neural Computing and Applications, vol. 32, no. 9, pp. 5185-5211, 2020.

[68] H. Haklı and H. Uğuz, "A novel particle swarm optimization algorithm with Levy flight," Applied Soft Computing, vol. 23, pp. 333-345, 2014.

[69] H. Zhang, J. Xie, Q. Hu, L. Shao, and T. Chen, "A hybrid DPSO with Levy flight for scheduling MIMO radar tasks," Applied Soft Computing, vol. 71, pp. 242-254, 2018.

[70] S. N. Chegini, A. Bagheri, and F. Najafi, "PSOSCALF: a new hybrid PSO based on Sine Cosine algorithm and Levy flight for solving optimization problems," Applied Soft Computing, vol. 73, pp. 697-726, 2018.

[71] R. Jensi and G. W. Jiji, "An enhanced particle swarm optimization with levy flight for global optimization," Applied Soft Computing, vol. 43, pp. 248-261, 2016.

[72] T. Easwarakhanthan, J. Bottin, I. Bouhouch, and C. Boutrit, "Nonlinear minimization algorithm for determining the solar cell parameters with microcomputers," International Journal of Solar Energy, vol. 4, no. 1, pp. 1-12, 1986.

[73] Z. Chen, L. Wu, P. Lin, Y. Wu, and S. Cheng, "Parameters identification of photovoltaic models using hybrid adaptive Nelder-Mead simplex algorithm based on eagle strategy," Applied Energy, vol. 182, pp. 47-57, 2016.

[74] X. Gao, Y. Cui, J. Hu et al., "Parameter extraction of solar cell models using improved shuffled complex evolution algorithm," Energy Conversion And Management, vol. 157, pp. 460-479, 2018.

[75] W. Huang, "Extracting solar cell model parameters based on chaos particle swarm algorithm," in Proceedings of the 2011 International Conference on Electric Information and Control Engineering, Wuhan, China, April 2011.

[76] F. Dkhichi, B. Oukarfi, A. Fakkar, and N. Belbounaguia, "Parameter identification of solar cell model using Levenberg-Marquardt algorithm combined with simulated annealing," Solar Energy, vol. 110, pp. 781-788, 2014.

[77] H. Chen, S. Jiao, M. Wang, A. A. Heidari, and X. Zhao, "Parameters identification of photovoltaic cells and modules using diversification-enriched Harris hawks optimization with chaotic driftse," Journal of Cleaner Production, vol. 244, Article ID 118778, 2020.

[78] X. Chen, K. Yu, W. Du, W. Zhao, and G. Liu, "Parameters identification of solar cell models using generalized oppositional teaching learning based optimization," Energy, vol. 99, pp. 170-180, 2016.

[79] D. Prasad, A. Mukherjee, G. Shankar, and V. Mukherjee, "Application of chaotic whale optimisation algorithm for transient stability constrained optimal power flow," IET Science, Measurement \& Technology, vol. 11, no. 8, pp. 1002-1013, 2017.

[80] K. M. El-Naggar, M. R. AlRashidi, M. F. AlHajri, and A. K. Al-Othman, "Simulated Annealing algorithm for photovoltaic parameters identification," Solar Energy, vol. 86, no. 1, pp. 266-274, 2012.

[81] A. Askarzadeh and A. Rezazadeh, "Parameter identification for solar cell models using harmony search-based algorithms," Solar Energy, vol. 86, no. 11, pp. 3241-3249, 2012.

[82] M. Chegaar, N. Nehaoua, and A. Bouhemadou, "Organic and inorganic solar cells parameters evaluation from single I-V plot," Energy Conversion and Management, vol. 49, no. 6, pp. 1376-1379, 2008.

[83] X. Yuan, Y. He, and L. Liu, "Parameter extraction of solar cell models using chaotic asexual reproduction optimization," Neural Computing and Applications, vol. 26, no. 5, pp. 1227-1239, 2015.

[84] M. Tubishat, M. A. M. Abushariah, N. Idris, and I. Aljarah, "Improved whale optimization algorithm for feature selection in Arabic sentiment analysis," Applied Intelligence, vol. 49, no. 5, pp. 1688-1707, 2019.

[85] Y. Wang, "Occupation recommendation based on student achievement mining in vocational skill training," in Proceedings of the 2014 11th International Conference on Fuzzy Systems and Knowledge Discovery (FSKD 2014), Xiamen, China, August 2014.

[86] S. Song, P. Wang, A. A. Heidari et al., "Dimension decided Harris hawks optimization with Gaussian mutation: balance analysis and diversity patterns," Knowledge-Based Systems, vol. 215, Article ID 106425, 2020.

[87] D. Zhao, L. Liu, F. Yu et al., "Ant colony optimization with horizontal and vertical crossover search: fundamental visions for multi-threshold image segmentation," Expert Systems with Applications, Article ID 114122, 2020, In press.

[88] D. Zhao, L. Liu, F. Yu et al., "Chaotic random spare ant colony optimization for multi-threshold image segmentation of 2D Kapur entropy," Knowledge-Based Systems, Article ID 106510, 2020, In press.

[89] Y. Zhang, R. Liu, A. A. Hedari et al., "Towards augmented kernel extreme learning models for Bankruptcy prediction: algorithmic behavior and comprehensive analysis," Neurocomputing, 2020, In press.

[90] X. Wang, H. Chen, A. A. Heidari et al., "Multi-population following behavior-driven fruit fly optimization: a Markov chain convergence proof and comprehensive analysis," Knowledge-Based Systems, vol. 210, Article ID 106437, 2020.

[91] J. Tu, H. Chen, J. Liu et al., "Evolutionary biogeographybased Whale optimization methods with communication structure: towards measuring the balance," Knowledge-Based Systems, Article ID 106642, 2020, In press.

[92] H. Chen, Y. Xu, M. Wang, and X. Zhao, "A balanced whale optimization algorithm for constrained engineering design problems," Applied Mathematical Modelling, vol. 71, pp. 45-59, 2019.

[93] M. A. Al-Betar, M. A. Awadallah, A. A. Heidari, H. Chen, H. Al-khraisat, and C. Li, "Survival exploration strategies for Harris Hawks optimizer," Expert Systems with Applications, Article ID 114243, 2020, In press.

[94] J. Luo, H. Chen, Q. Zhang, and Y. Xu, “An improved grasshopper optimization algorithm with application to financial stress prediction," Applied Mathematical Modelling, vol. 64, pp. 654-668, 2018. 
[95] H. Chen, M. Wang, and X. Zhao, "A multi-strategy enhanced sine cosine algorithm for global optimization and constrained practical engineering problems," Applied Mathematics and Computation, vol. 369, Article ID 124872, 2020.

[96] H. Chen, S. Li, A. Asghar Heidari et al., "Efficient multipopulation outpost fruit fly-driven optimizers: framework and advances in support vector machines," Expert Systems with Applications, vol. 142, Article ID 112999, 2020.

[97] L. Shen, H. Chen, Z. Yu et al., "Evolving support vector machines using fruit fly optimization for medical data classification," Knowledge-Based Systems, vol. 96, pp. 61-75, 2016.

[98] M. Wang, H. Chen, B. Yang et al., “Toward an optimal kernel extreme learning machine using a chaotic moth-flame optimization strategy with applications in medical diagnoses," Neurocomputing, vol. 267, pp. 69-84, 2017.

[99] J. Xia, H. Chen, Q. Li et al., "Ultrasound-based differentiation of malignant and benign thyroid Nodules: an extreme learning machine approach," Computer Methods and Programs in Biomedicine, vol. 147, pp. 37-49, 2017.

[100] H.-L. Chen, G. Wang, C. Ma, Z.-N. Cai, W.-B. Liu, and S.-J. Wang, "An efficient hybrid kernel extreme learning machine approach for early diagnosis of Parkinson's disease," Neurocomputing, vol. 184, pp. 131-144, 2016.

[101] L. Hu, G. Hong, J. Ma, X. Wang, and H. Chen, “An efficient machine learning approach for diagnosis of paraquat-poisoned patients," Computers in Biology and Medicine, vol. 59, pp. 116-124, 2015.

[102] S.-J. Wang, H.-L. Chen, W.-J. Yan, Y.-H. Chen, and X. Fu, "Face recognition and micro-expression recognition based on discriminant tensor subspace analysis plus extreme learning machine," Neural Processing Letters, vol. 39, no. 1, pp. 25-43, 2014.

[103] X. Zhao, X. Zhang, Z. Cai et al., "Chaos enhanced grey wolf optimization wrapped ELM for diagnosis of paraquat-poisoned patients," Computational Biology and Chemistry, vol. 78, pp. 481-490, 2019.

[104] X. Zhang, T. Wang, J. Wang, and G. Tang, "Pyramid channel-based feature attention network for image dehazing," Computer Vision and Image Understanding, pp. 197-198, Article ID 103003, 2020.

[105] Y. Li, W.-G. Cui, H. Huang, Y.-Z. Guo, K. Li, and T. Tan, "Epileptic seizure detection in EEG signals using sparse multiscale radial basis function networks and the Fisher vector approach," Knowledge-Based Systems, vol. 164, pp. 96-106, 2019.

[106] Y. Li, J. Liu, Z. Tang, and B. Lei, "Deep spatial-temporal feature fusion from adaptive dynamic functional connectivity for MCI identification," IEEE Transactions on Medical Imaging, vol. 39, no. 9, pp. 2818-2830, 2020.

[107] W. Deng, H. Liu, J. Xu, and H. Zhao, "An improved quantum-inspired differential evolution algorithm for deep belief network," IEEE Transactions on Instrumentation \& Measurement, vol. 99, no. 1, 2020.

[108] D. Liu, S. Wang, D. Huang, G. Deng, F. Zeng, and H. Chen, "Medical image classification using spatial adjacent histogram based on adaptive local binary patterns," Computers in Biology and Medicine, vol. 72, pp. 185-200, 2016.

[109] X. Zhang, Y. Xu, C. Yu et al., "Gaussian mutational chaotic fruit fly-built optimization and feature selection," Expert Systems with Applications, vol. 141, Article ID 112976, 2020.

[110] Y. Zhang, R. Liu, X. Wang, H. Chen, and C. Li, "Boosted binary Harris hawks optimizer and feature selection," Engineering with Computers, 2020, In press.
[111] X. Zhao, D. Li, B. Yang, C. Ma, Y. Zhu, and H. Chen, "Feature selection based on improved ant colony optimization for online detection of foreign fiber in cotton," Applied Soft Computing, vol. 24, pp. 585-596, 2014.

[112] X. Zhao, D. Li, B. Yang et al., "A two-stage feature selection method with its application," Computers \& Electrical Engineering, vol. 47, pp. 114-125, 2015.

[113] C. Li, L. Hou, B. Y. Sharma et al., "Developing a new intelligent system for the diagnosis of tuberculous pleural effusion," Computer Methods and Programs in Biomedicine, vol. 153, pp. 211-225, 2018.

[114] Z. Chen, M. Lu, Y. Zhou, and C. Chen, "Information synergy entropy based multi-feature information fusion for the operating condition identification in aluminium electrolysis," Information Sciences, vol. 548, pp. 275-294, 2021.

[115] X. Xue, S. Wang, L. Zhang, Z. Feng, and Y. Guo, "Social learning evolution (SLE): computational experiment-based modeling framework of social manufacturing," IEEE Transactions on Industrial Informatics, vol. 15, no. 6, pp. 3343-3355, 2019

[116] D. Wang, Y. Liang, D. Xu, X. Feng, and R. Guan, "A contentbased recommender system for computer science publications," Knowledge-Based Systems, vol. 157, pp. 1-9, 2018.

[117] R. Guan, H. Zhang, Y. Liang, F. Giunchiglia, L. Huang, and $\mathrm{X}$. Feng, "Deep feature-based text clustering and its explanation," IEEE Transactions on Knowledge and Data Engineering, p. 1, 2020, In press.

[118] C. Yang, L. Bruzzone, H. Zhao, Y. Tan, and R. Guan, "Superpixel-based unsupervised band selection for classification of hyperspectral images," IEEE Transactions on Geoscience and Remote Sensing, vol. 56, no. 12, pp. 7230 7245, 2018. 